\title{
Solvability of BVPs for impulsive fractional differential equations involving the Riemann-Liouville fractional derivatives
}

\author{
Yuji Liu
}

\begin{abstract}
In this paper, we study two classes of BVPs for impulsive fractional differential equations. Some existence results for these boundary value problems are established. Some comments on three published papers are made.
\end{abstract}

Mathematics Subject Classification (2010): 34K37, 34K45, 34B37, 34B15, 34B10, 92D25, 34A37, 34K15.

Keywords: Impulsive fractional differential equation, boundary value problem, Riemann-Liouville fractional derivative.

\section{Introduction}

Impulsive fractional differential equations is an important area of study [1]. In recent years, boundary value problems (BVPs) or initial value problems (IVPs) for impulsive fractional differential equations (IFDEs) have been studied by many authors. For example, in $[2,4,3,9,11,14,15]$, the authors studied the existence or uniqueness of solutions of BVPs for IFDEs with Caputo type fractional derivatives and multiple starting points.

In [8], Kosmatov studied the following problem:

$$
\left\{\begin{array}{l}
D_{0^{+}}^{\alpha} x(t)=f(t, x(t)), t \in[0,1] \backslash\left\{t_{1}, t_{2}, \cdots, t_{m}\right\}, \\
D_{0^{+}}^{\beta} x\left(t_{k}^{+}\right)-D_{+}^{\beta} x\left(t_{k}^{-}\right)=J_{k}\left(x\left(t_{k}\right)\right), i=1,2, \cdots, m, I_{0^{+}}^{1-\alpha} x(0)=x_{0},
\end{array}\right.
$$

where $\alpha \in(0,1), D_{0^{+}}^{*}$ is the standard Riemann-Liouville fractional derivative of order $*, \beta \in(0, \alpha), x_{0} \in \mathbb{R}, 0=t_{0}<t_{1}<\cdots<t_{m}<t_{m+1}=1, J_{k}: \mathbb{R} \rightarrow \mathbb{R}, f:[0,1] \times \mathbb{R} \rightarrow$ $\mathbb{R}$ are suitable functions.

The author was supported by the Natural Science Foundation of Guangdong province (No: S2011010001900) and the Natural science research project for colleges and universities of Guangdong Province (No: 2014KTSCX126). 
In [9], Liu studied the solvability of two classes of initial value problems of nonlinear impulsive multi-term fractional differential equations on half lines. One (IVP (1) for short) is as follows:

$$
\left\{\begin{array}{l}
D_{0^{+}}^{\alpha} x(t)+q(t) f\left(t, x(t), D_{0^{+}}^{p} x(t)\right)=0, \quad t \in\left(t_{i}, t_{i+1}\right), i \in \mathbf{N}_{0}^{m}, \\
\lim _{t \rightarrow 0^{+}} t^{1-\alpha} x(t)=x_{0}, \\
\left.\lim _{t \rightarrow t_{i}^{+}}\left(t-t_{i}\right)^{1-\alpha} x(t)=I\left(t_{i}, x\left(t_{i}\right), D_{0^{+}}^{p} x\left(t_{i}\right)\right)\right), i \in \mathbf{N}_{1}^{m},
\end{array}\right.
$$

and

$$
\left\{\begin{array}{l}
D_{t_{i}^{+}}^{\alpha} x(t)+q_{1}(t) f_{1}\left(t, x(t), D_{t_{i}^{+}}^{p} x(t)\right)=0, \quad t \in\left(t_{i}, t_{i+1}\right), i \in \mathbf{N}_{0}^{m}, \\
\lim _{t \rightarrow 0^{+}} t^{1-\alpha} x(t)=x_{0} \\
\lim _{t \rightarrow t_{i}^{+}}\left(t-t_{i}\right)^{1-\alpha} x(t)=I_{1}\left(t_{i}, x\left(t_{i}\right), D_{t_{i-1}^{+}}^{p} x\left(t_{i}\right)\right), i \in \mathbf{N}_{1}^{m}
\end{array}\right.
$$

where $\alpha \in(0,1), 0<p<\alpha, D_{a^{+}}^{b}$ is the standard Riemann-Liouville fractional derivative of order $b>0$ with starting point $a, x_{0} \in \mathbf{R}, \mathbf{N}_{0}{ }^{m}=\{0,1,2, \cdots, m\}$ and $\mathbf{N}_{1}^{m}=\{1,2,3, \cdots, m\}, 0=t_{0}<t_{1}<t_{2}<t_{3}<\cdots<t_{m}<t_{m+1}=1, q:(0,1) \mapsto \mathbf{R}$ is continuous and satisfies that there exists $l \in(-1,-\alpha)$ such that $|q(t)| \leq t^{l}$ for all $t \in(0,1), q_{1}: \bigcup_{i=0}^{m}\left(t_{i}, t_{i+1}\right) \mapsto \mathbf{R}$ is continuous and satisfies that there exists $k_{1}>-1, l_{1} \leq 0$ such that $\left|q_{1}(t)\right| \leq\left(t-t_{i}\right)^{k_{1}}\left(t_{i+1}-t\right)^{l_{1}}$ for all $t \in\left(t_{i}, t_{i+1}\right)\left(i \in \mathbf{N}_{0}\right)$, $f:(0,1) \times \mathbf{R}^{2} \mapsto \mathbf{R}$ is a I-Carathéodory function, $f_{1}:\left(\bigcup_{i=0}^{m}\left(t_{i}, t_{i+1}\right)\right) \times \mathbf{R}^{2} \mapsto \mathbf{R}$ is a II-Carathéodory function, $I, I_{1}:\left\{t_{i}: i \in \mathbf{N}\right\} \times \mathbf{R}^{2} \mapsto \mathbf{R}$ are discrete Carathéodory functions. IFDE

In [14], the authors studied the existence of solutions of the following BVP for

$$
\left\{\begin{array}{l}
D_{0^{+}}^{q} x(t)+\lambda(t) x(t)=f(t, x(t)), t \in[0,1] \backslash\left\{t_{1}, t_{2}, \cdots, t_{m}\right\}, \\
I_{0^{+}}^{\alpha} x\left(t_{i}^{+}\right)-I_{0^{+}}^{\alpha}\left(t_{i}^{-}\right)=J_{i}\left(x\left(t_{i}\right)\right), i=1,2, \cdots, m,\left.t^{1-q} x(t)\right|_{t=0}+\left.t^{1-q} x(t)\right|_{t=1}=0,
\end{array}\right.
$$

where $q, \alpha \in(0,1), D_{0^{+}}^{q}$ is the Riemann-Liouville fractional derivative, $I_{0^{+}}^{\alpha}$ is the Riemann-Liouville fractional integral, $0=t_{0}<t_{1}<\cdots<t_{m}<t_{m+1}=1, \lambda \in$ $C^{0}([0,1], \mathbb{R})$ satisfies $\lambda_{0}=: \max _{t \in[0,1]} \lambda(t)>0, J_{k}: \mathbf{R} \mapsto \mathbf{R}$ is continuous, $f$ is a given piecewise continuous function. The following special case play a large role in the proof of the main theorem:

$$
\left\{\begin{array}{l}
D_{0^{+}}^{q} x(t)+\lambda_{0} x(t)=f(t, x(t)), t \in[0,1] \backslash\left\{t_{1}, t_{2}, \cdots, t_{m}\right\}, \\
I_{0^{+}}^{\alpha} x\left(t_{i}^{+}\right)-I_{0^{+}}^{\alpha}\left(t_{i}^{-}\right)=J_{i}\left(x\left(t_{i}\right)\right), i=1,2, \cdots, m,\left.t^{1-q} x(t)\right|_{t=0}+\left.t^{1-q} x(t)\right|_{t=1}=0
\end{array}\right.
$$


In [17], Zhao studied the following higher-order nonlinear Riemann-Liouville fractional differential equation with Riemann-Stieltjes integral boundary value conditions and impulses

$$
\left\{\begin{array}{l}
-D_{0^{+}}^{\alpha} x(t)=\lambda a(t) f(t, x(t)), t \backslash\left\{t_{i}\right\}_{i=1}^{m}, \\
\Delta x\left(t_{i}\right)=I_{i}\left(x\left(t_{i}\right)\right), i=1,2, \cdots, m, \\
x(0)=x^{\prime}(0)=\cdots=x^{(n-2)}(0)=0, x^{\prime}(1)=\int_{0}^{1} x(s) d H(s),
\end{array}\right.
$$

where $D_{0^{+}}^{\alpha}$ is the standard Riemann-Liouville fractional derivative of order $n-1<$ $\alpha \leq n$ with $n \geq 3$, the impulsive point sequence $0=t_{0}<t_{1}<t_{2}<\cdots<t_{m}<$ $t_{m+1}=1, \lambda>0, f \in C([0,1] \times[0,+\infty),[0,+\infty)), a \in C((0,1),[0,+\infty))$, the integral $\int_{0}^{1} x(s) d H(s)$ is the Riemann-Stieltjes integral with $H:[0,1] \rightarrow \mathbb{R}$ with

$$
\delta=: \int_{0}^{1} s^{\alpha-1} d H(s) \neq \alpha-1 .
$$

Motivated by $[8,17]$, we investigate the solvability of the following two boundary value problems for impulsive fractional differential equations

$$
\left\{\begin{array}{l}
D_{0^{+}}^{\alpha} x(t)-\lambda x(t)=f(t, x(t)), a . e ., t \in\left(t_{i}, t_{i+1}\right], i \in \mathbb{N}_{0}^{m}, \\
\Delta I_{0^{+}}^{\beta} x\left(t_{i}\right)=: I_{0^{+}}^{\beta} x\left(t_{i}^{+}\right)-I_{0^{+}}^{\beta} x\left(t_{i}\right)=I_{n}\left(t_{i}, x\left(t_{i}\right)\right), i \in \mathbb{N}_{1}^{m}, \\
\Delta D_{0^{+}}^{\alpha-j} x\left(t_{i}\right)=: D_{0^{+}}^{\alpha-j} x\left(t_{i}^{+}\right)-D_{0^{+}}^{\alpha-j} x\left(t_{i}\right)=I_{j}\left(t_{i}, x\left(t_{i}\right)\right), i \in \mathbb{N}_{1}^{m}, j \in \mathbb{N}_{1}^{n-1}, \\
I_{0^{+}}^{n-\alpha} x(0)=x_{n}, D_{0^{+}}^{\alpha-j} x(0)=x_{j}, j \in \mathbb{N}_{1}^{n-1}
\end{array}\right.
$$

and

$$
\left\{\begin{array}{l}
D_{0^{+}}^{\alpha} x(t)=f(t, x(t)), t \backslash\left\{t_{i}\right\}_{i=1}^{m}, \\
\Delta D_{0^{+}}^{\alpha-j} x\left(t_{i}\right)=I_{j}\left(t_{i}, x\left(t_{i}\right)\right), i \in \mathbb{N}_{1}^{m}, j \in \mathbb{N}_{1}^{n-1} \\
\Delta I_{0^{+}}^{n-\alpha} x\left(t_{i}=I_{n}\left(t_{i}, x\left(t_{i}\right)\right), i \in \mathbb{N}_{1}^{m}\right. \\
I_{0^{+}}^{n-\alpha} x(0)=D_{0^{+}}^{\alpha-j} x(0)=0, j \in \mathbb{N}_{1}^{n-2}, D_{0^{+}}^{\alpha-n+1} x(1)=\int_{0}^{1} x(s) d H(s),
\end{array}\right.
$$

where $m, n$ are positive integers, $\alpha \in(n-1, n), \beta>0, \lambda \in \mathbb{R}, 0=t_{0}<t_{1}<\cdots<$ $t_{m}<t_{m+1}=1, x_{j} \in \mathbb{R}\left(j \in \mathbb{N}_{1}^{n}, f:(0,1) \times \mathbb{R} \rightarrow \mathbb{R}\right.$ is a Carathéodory fraction, $I_{j}:\left\{t_{i}: i \in \mathbb{N}_{1}^{m}\right\} \times \mathbb{R} \rightarrow \mathbb{R}$ is a discrete Carathéodory function $\left(j \in \mathbb{N}_{1}^{n}\right)$.

The purposes of this paper are to establish existence results for solutions of IVP (1.7) $(\alpha-\beta-n=0)$ and existence results for solutions of $\operatorname{BVP}(1.8)$ respectively. The method used is based upon the fixed point theorems. The results in this paper complement known ones in [8, 17] and generalize known ones [10]. 
A function $x:(0,1] \rightarrow \mathbb{N}$ is called a solution of IVP (1.7) (or IVP (1.8)) if $x \in C\left(t_{i}, t_{i+1}\right], \lim _{t \rightarrow t_{i}^{+}}\left(t-t_{i}\right)^{n-\alpha} x(t)$ is finite for $i \in \mathbb{N}_{0}^{m}$ and $\left.D_{0^{+}}^{\alpha} x\right|_{\left(t_{i}, t_{i+1}\right]}-\left.\lambda x\right|_{\left(t_{i}, t_{i+1}\right]} \in$ $L^{1}\left(t_{i}, t_{i+1}\right)$, and $x$ satisfies all equations in (1.6) (or (1.8)).

The remainder of this paper is divided into three sections. In Section 2, we present related definitions and preliminary results. In Section 3, we establish existence results for IVP (1.7) and BVP(1.8) respectively. In Section 4, we give comments on some published papers.

\section{Preliminary results}

For the convenience of the readers, we firstly present the necessary definitions from the fractional calculus theory. These definitions can be found in the literature $[5,6,7]$.

Let $a<b$. Denote $L^{1}(a, b)$ the set of all integrable functions on $(a, b), C^{0}(a, b]$ the set of all continuous functions on $(a, b]$. For $\phi \in L^{1}(a, b)$, denote

$$
\|\phi\|_{1}=\int_{a}^{b}|\phi(s)| d s .
$$

For $\phi \in C^{0}[a, b]$, denote $\|\phi\|_{0}=\max _{t \in[a, b]}|\phi(t)|$.

For two integers $a<b$, denote $\mathbb{N}_{a}^{b}=\{a, a+1, \cdots, b\}$.

Let the Gamma and beta functions $\Gamma(\alpha), \mathbf{B}(p, q)$ and the Mitag-Leffler function $\mathbf{E}_{\alpha, \delta}(x)$ be defined by

$$
\begin{gathered}
\Gamma(\alpha)=\int_{0}^{+\infty} x^{\alpha-1} e^{-x} d x \\
\mathbf{B}(p, q)=\int_{0}^{1} x^{p-1}(1-x)^{q-1} d x, \\
\mathbf{E}_{\alpha, \delta}(x)=\sum_{k=0}^{\infty} \frac{x^{k}}{\Gamma(k \alpha+\delta)}, \alpha, p, q, \delta>0 .
\end{gathered}
$$

Definition 2.1. The Riemann-Liouville fractional integral of order $\alpha>0$ of a function $g:(0, \infty) \mapsto \mathbf{R}$ (may be piecewise continuous) is given by

$$
I_{0^{+}}^{\alpha} g(t)=\frac{1}{\Gamma(\alpha)} \int_{0}^{t}(t-s)^{\alpha-1} g(s) d s,
$$

provided that the right-hand side exists.

Definition 2.2. The Riemann-Liouville fractional derivative of order $\alpha>0$ of a function $g:(0, \infty) \mapsto \mathbf{R}$ (may be piecewise continuous) is given by

$$
D_{0^{+}}^{\alpha} g(t)=\frac{1}{\Gamma(n-\alpha)} \frac{d^{n}}{d t^{n}} \int_{0}^{t} \frac{g(s)}{(t-s)^{\alpha-n+1}} d s,
$$

where $n-1<\alpha<n$, provided that the right-hand side exists.

Remark 2.1. For a piecewise continuous function $g$ which is continuous on $\left(t_{i}, t_{i+1}\right]$ $\left(i \in \mathbf{N}_{0}^{m}, 0=t_{0}<t_{1}<\cdots<t_{i}<\cdots<t_{m}<t_{m+1}=1\right)$, and $t \in\left(t_{i}, t_{i+1}\right]$, the 
Riemann-Liouville fractional integral of order $\alpha>0$ of $g$ on $(0, t]$ with $t \in\left(t_{i}, t_{i+1}\right]$ is given by

$$
\begin{aligned}
I_{0^{+}}^{\alpha} g(t) & =\frac{1}{\Gamma(\alpha)} \int_{0}^{t}(t-s)^{\alpha-1} g(s) d s \\
& =\sum_{j=0}^{i} \int_{t_{j}}^{t_{j+1}} \frac{(t-s)^{\alpha-1} g(s) d s}{\Gamma(\alpha)}+\int_{t_{i}}^{t} \frac{(t-s)^{\alpha-1} g(s) d s}{\Gamma(\alpha)},
\end{aligned}
$$

provided that each term in the right-hand side exists.

Let $\alpha \in(n-1, n)$ with $n$ being a positive integer. For a piecewise continuous function $g$ which is continuous on $\left(t_{i}, t_{i+1}\right]\left(i \in \mathbf{N}_{0}^{m}, 0=t_{0}<t_{1}<\cdots<t_{i}<\cdots<\right.$ $\left.t_{m}<t_{m+1}=1\right)$, and $t \in\left(t_{i}, t_{i+1}\right]$, the Riemann-Liouville fractional derivative of order $\alpha>0$ of $g$ on $(0, t]$ with $t \in\left(t_{i}, t_{i+1}\right]$ is given by

$$
\begin{aligned}
D_{0^{+}}^{\alpha} g(t) & =\frac{1}{\Gamma(n-\alpha)}\left[\int_{0}^{t}(t-s)^{n-\alpha-1} g(s) d s\right]^{(n)} \\
& =\frac{\left[\sum_{j=0}^{i} \int_{t_{j}}^{t_{j+1}}(t-s)^{n-\alpha-1} g(s) d s+\int_{t_{i}}^{t}(t-s)^{n-\alpha-1} g(s) d s\right]^{(n)}}{\Gamma(n-\alpha)}
\end{aligned}
$$

provided that each term in the right-hand side exists.

Definition 2.3. We call $F:(0,1) \times \mathbf{R} \mapsto \mathbf{R}$ a Carathéodory function if it satisfies the followings:

(i) $t \mapsto F\left(t,\left(t-t_{i}\right)^{n-\alpha} u\right)$ are measurable on $\left(t_{i}, t_{i+1}\right)\left(i \in \mathbf{N}_{0}^{m}\right)$ for any $u \in \mathbf{R}$,

(ii) $u \mapsto F\left(t,\left(t-t_{i}\right)^{n-\alpha} u\right)$ is continuous on $\mathbf{R}$ for all $t \in\left(t_{i}, t_{i+1}\right)\left(i \in \mathbf{N}_{0}^{m}\right)$,

(iii) for each $r>0$, there exists $M_{r} \geq 0$ such that $\left|F\left(t,\left(t-t_{i}\right)^{n-\alpha} u\right)\right| \leq M_{r}$ for all $t \in\left(t_{i}, t_{i+1}\right)\left(i \in \mathbf{N}_{0}^{m}\right)$ and $|u| \leq r$.

Definition 2.4. We call $G:\left\{t_{i}: i \in \mathbf{N}_{1}^{m}\right\} \times \mathbf{R} \mapsto \mathbf{R}$ a discrete I-Carathéodory function if it satisfies the followings:

(i) $u \mapsto G\left(t_{i},\left(t_{i}-t_{i-1}\right)^{n-\alpha} u\right)$ is continuous on $\mathbf{R}$ for all $i \in \mathbf{N}_{1}^{m}$,

(ii) for each $r>0$, there exists $M_{r} \geq 0$ such that $\left|G\left(t_{i},\left(t_{i}-t_{i-1}\right)^{n-\alpha} u\right)\right| \leq M_{r}$ for all $i \in \mathbf{N}_{1}^{m}$ and $|u| \leq r$.

Suppose that $\alpha \in(n-1, n), 0=t_{0}<t_{1}<\cdots<t_{m+1}=1$. Denote

$$
P C_{n-\alpha}(0, T]=\left\{x:(0,1] \mapsto \mathbf{R}: \begin{array}{c}
\left.x\right|_{\left(t_{i}, t_{i+1}\right]} \in C^{0}\left(t_{i}, t_{i+1}\right], i \in \mathbf{N}_{0}^{m}, \\
\lim _{t \rightarrow t_{i}^{+}}\left(t-t_{i}\right)^{n-\alpha} x(t), i \in \mathbf{N}_{0}^{m} \text { are finite }
\end{array}\right\} .
$$

Define

$$
\|x\|=\|x\|_{n-\alpha}=\max \left\{\sup _{t \in\left(t_{i}, t_{i+1}\right]}\left(t-t_{i}\right)^{n-\alpha}|x(t)|, i \in \mathbf{N}_{0}^{m}\right\} .
$$

Then $P C_{n-\alpha}$ is a Banach space with the norm $\|\cdot\|$ defined.

Theorem 2.1. Suppose that $\alpha \in(n-1, n), \lambda \in \mathbb{R}, h:(0,1) \rightarrow \mathbb{R}$ is continuous and satisfies $|h(t)| \leq t^{k}(1-t)^{l}$ for all $t \in(0,1)$, where $k>-1, l \leq 0$ with $1+k+l>0$. 
Then $x \in P C_{n-\alpha}(0,1]$ is a piecewise continuous solution of

$$
D_{0^{+}}^{\alpha} x(t)-\lambda x(t)=h(t) \text {, a.e., } t \in\left(t_{i}, t_{i+1}\right], i \in \mathbb{N}_{0}^{m}
$$

if and only if there exist constants $c_{\nu k} \in \mathbb{R}\left(\nu \in \mathbb{N}_{1}^{n}, k \in \mathbb{N}_{0}\right)$ such that

$$
\begin{aligned}
l x(t) & =\int_{0}^{t}(t-s)^{\alpha-1} \mathbf{E}_{\alpha, \alpha}\left(\lambda(t-s)^{\alpha}\right) h(s) d s \\
& +\sum_{k=0}^{j} \sum_{\nu=1}^{n} c_{\nu k}\left(t-t_{k}\right)^{\alpha-\nu} \mathbf{E}_{\alpha, \alpha-\nu+1}\left(\lambda\left(t-t_{k}\right)^{\alpha}\right), t \in\left(t_{j}, t_{j+1}\right], j \in \mathbb{N}_{0}^{m} .
\end{aligned}
$$

Proof. We have

$$
\begin{gathered}
t^{n-\alpha}\left|\int_{0}^{t}(t-s)^{\alpha-1} \mathbf{E}_{\alpha, \alpha}\left(\lambda(t-s)^{\alpha}\right) h(s) d s\right| \\
\leq t^{n-\alpha} \sum_{\chi=0}^{\infty} \frac{|\lambda| \chi}{\Gamma(\chi \alpha+\alpha)} \int_{0}^{t}(t-s)^{\alpha-1+\chi \alpha} s^{k}(1-s)^{l} d s \\
=t^{n-\alpha} \sum_{\chi=0}^{\infty} \frac{|\lambda| \chi}{\Gamma(\chi \alpha+\alpha)} t^{\chi \alpha+\alpha} \int_{0}^{1}(1-w)^{\alpha+l-1+\chi \alpha} w^{k} d w \\
\leq t^{n-\alpha} \sum_{\chi=0}^{\infty} \frac{|\lambda|^{\chi}}{\Gamma(\chi \alpha+\alpha)} t^{\chi \alpha+k+\alpha+l} \int_{0}^{1}(1-w)^{\alpha+l-1} w^{k} d w \\
=\mathbf{B}(\alpha+l, k+1) \mathbf{E}_{\alpha, \alpha}\left(|\lambda| t^{\alpha}\right) t^{n+k+l} \rightarrow 0 \text { as } t \rightarrow 0 .
\end{gathered}
$$

Then $t \rightarrow t^{n-\alpha} \int_{0}^{t}(t-s)^{\alpha-1} \mathbf{E}_{\alpha, \alpha}\left(\lambda(t-s)^{\alpha}\right) h(s) d s$ is continuous on $[0,1]$.

Step 1. Assume $x \in P C_{n-\alpha}(0,1]$ is a solution of (2.1). We prove $x$ satisfies (2.2).

From (5.1)-(5.3) in [7], there exist constants $c_{\nu 0} \in \mathbb{R}$ such that

$$
x(t)=\int_{0}^{t}(t-s)^{\alpha-1} \mathbf{E}_{\alpha, \alpha}\left(\lambda(t-s)^{\alpha}\right) h(s) d s+\sum_{\nu=1}^{n} c_{\nu 0} t^{\alpha-\nu} \mathbf{E}_{\alpha, \alpha-\nu+1}\left(\lambda t^{\alpha}\right), t \in\left(t_{0}, t_{1}\right] .
$$

It follows that (2.2) holds for $j=0$. Now suppose that (3.7) holds for $i=0,1, \cdots, \omega<$ $m$, i.e.,

$$
\begin{aligned}
x(t) & =\int_{0}^{t}(t-s)^{\alpha-1} \mathbf{E}_{\alpha, \alpha}\left(\lambda(t-s)^{\alpha}\right) h(s) d s \\
& +\sum_{k=0}^{j} \sum_{\nu=1}^{n} c_{\nu k}\left(t-t_{k}\right)^{\alpha-\nu} \mathbf{E}_{\alpha, \alpha-\nu+1}\left(\lambda\left(t-t_{k}\right)^{\alpha}\right), t \in\left(t_{j}, t_{j+1}\right], j \in \mathbb{N}_{0}^{\omega} .
\end{aligned}
$$

We will prove that (2.2) holds for $i=\omega+1$. Then by mathematical induction method, (2.2) holds for all $i \in \mathbb{N}_{0}^{m}$. In order to get the exact expression of $x$ on $\left(t_{\omega+1}, t_{\omega+2}\right]$, we suppose that there exists $\Phi$ such that

$$
\begin{aligned}
x(t) & =\Phi(t)+\int_{0}^{t}(t-s)^{\alpha-1} \mathbf{E}_{\alpha, \alpha}\left(\lambda(t-s)^{\alpha}\right) h(s) d s \\
& +\sum_{k=0}^{\omega} \sum_{\nu=1}^{n} c_{\nu k}\left(t-t_{k}\right)^{\alpha-\nu} \mathbf{E}_{\alpha, \alpha-\nu+1}\left(\lambda\left(t-t_{k}\right)^{\alpha}\right), t \in\left(t_{\omega+01}, t_{\omega+2}\right] .
\end{aligned}
$$


Solvability of BVPs for impulsive fractional differential equations

Using Definition 2.2, (2.3) and (2.4), we know for $t \in\left(t_{\omega+1}, t_{\omega+2}\right]$ by direct computation that

$$
\begin{aligned}
& D_{0^{+}}^{\alpha} x(t)=\frac{\left[\int_{0}^{t}(t-s)^{n-\alpha-1} x(s) d s\right]^{(n)}}{\Gamma(n-\alpha)} \\
& =\frac{\left[\sum_{\rho=0}^{\omega} \int_{t_{\rho}}^{t_{\rho}+1}(t-s)^{n-\alpha-1} x(s) d s+\int_{t_{\omega+1}}^{t}(t-s)^{n-\alpha-1} x(s) d s\right]^{(n)}}{\Gamma(n-\alpha)} \\
& =\left[\sum _ { \rho = 0 } ^ { \omega } \int _ { t _ { \rho } } ^ { t _ { \rho } + 1 } ( t - s ) ^ { n - \alpha - 1 } \left(\int_{0}^{s}(s-u)^{\alpha-1} \mathbf{E}_{\alpha, \alpha}\left(\lambda(s-u)^{\alpha}\right) h(u) d u\right.\right. \\
& \left.\left.+\sum_{k=0}^{\rho} \sum_{\nu=1}^{n} c_{\nu k}\left(s-t_{k}\right)^{\alpha-\nu} \mathbf{E}_{\alpha, \alpha-\nu+1}\left(\lambda\left(s-t_{k}\right)^{\alpha}\right)\right) d s\right]^{(n)} / \Gamma(n-\alpha) \\
& +\left[\int _ { t _ { \omega + 1 } } ^ { t } ( t - s ) ^ { n - \alpha - 1 } \left(\Phi(s)+\int_{0}^{s}(s-u)^{\alpha-1} \mathbf{E}_{\alpha, \alpha}\left(\lambda(s-u)^{\alpha}\right) h(u) d u\right.\right. \\
& \left.\left.+\sum_{k=0}^{\omega} \sum_{\nu=1}^{n} c_{\nu k}\left(s-t_{k}\right)^{\alpha-\nu} \mathbf{E}_{\alpha, \alpha-\nu+1}\left(\lambda\left(s-t_{k}\right)^{\alpha}\right)\right) d s\right]^{(n)} / \Gamma(n-\alpha) \\
& =D_{t_{\omega+1}^{+}}^{\alpha} \Phi(t) \\
& +\frac{\left[\sum_{\rho=0}^{\omega} \sum_{k=0}^{\rho} \sum_{\nu=1}^{n} c_{\nu k} \int_{t_{\rho}}^{t_{\rho}+1}(t-s)^{n-\alpha-1}\left(s-t_{k}\right)^{\alpha-\nu} \mathbf{E}_{\alpha, \alpha-\nu+1}\left(\lambda\left(s-t_{k}\right)^{\alpha}\right) d s\right]^{(n)}}{\Gamma(n-\alpha)} \\
& +\left[\int_{0}^{t}(t-s)^{n-\alpha-1} \int_{0}^{s}(s-u)^{\alpha-1} \mathbf{E}_{\alpha, \alpha}\left(\lambda(s-u)^{\alpha}\right) h(u) d u d s\right. \\
& \left.+\sum_{k=0}^{\omega} \sum_{\nu=1}^{n} c_{\nu k} \int_{t_{\omega+1}}^{t}(t-s)^{n-\alpha-1}\left(s-t_{k}\right)^{\alpha-\nu} \mathbf{E}_{\alpha, \alpha-\nu+1}\left(\lambda\left(s-t_{k}\right)^{\alpha}\right) d s\right]^{(n)} / \Gamma(n-\alpha) \\
& =D_{t_{\omega+1}^{+}}^{\alpha} \Phi(t) \\
& +\frac{\left[\sum_{k=0}^{\omega} \sum_{\rho=k}^{\omega} \sum_{\nu=1}^{n} c_{\nu k} \int_{t_{\rho}}^{t_{\rho}+1}(t-s)^{n-\alpha-1}\left(s-t_{k}\right)^{\alpha-\nu} \mathbf{E}_{\alpha, \alpha-\nu+1}\left(\lambda\left(s-t_{k}\right)^{\alpha}\right) d s\right]^{(n)}}{\Gamma(n-\alpha)} \\
& +\left[\int_{0}^{t}(t-s)^{n-\alpha-1} \int_{0}^{s}(s-u)^{\alpha-1} \mathbf{E}_{\alpha, \alpha}\left(\lambda(s-u)^{\alpha}\right) h(u) d u d s\right. \\
& \left.+\sum_{k=0}^{\omega} \sum_{\nu=1}^{n} c_{\nu k} \int_{t_{\omega+1}}^{t}(t-s)^{n-\alpha-1}\left(s-t_{k}\right)^{\alpha-\nu} \mathbf{E}_{\alpha, \alpha-\nu+1}\left(\lambda\left(s-t_{k}\right)^{\alpha}\right) d s\right]^{(n)} / \Gamma(n-\alpha)
\end{aligned}
$$




$$
\begin{aligned}
= & D_{t_{\omega+1}^{+}}^{\alpha} \Phi(t)+\frac{\left[\int_{0}^{t}(t-s)^{n-\alpha-1} \int_{0}^{s}(s-u)^{\alpha-1} \mathbf{E}_{\alpha, \alpha}\left(\lambda(s-u)^{\alpha}\right) h(u) d u d s\right]^{(n)}}{\Gamma(n-\alpha)} \\
& +\frac{\left[\sum_{k=0}^{\omega} \sum_{\nu=1}^{n} c_{\nu k} \int_{t_{k}}^{t}(t-s)^{n-\alpha-1}\left(s-t_{k}\right)^{\alpha-\nu} \mathbf{E}_{\alpha, \alpha-\nu+1}\left(\lambda\left(s-t_{k}\right)^{\alpha}\right) d s\right]^{(n)}}{\Gamma(n-\alpha)} \\
= & D_{t_{\omega+1}^{+}}^{\alpha} \Phi(t)+\frac{\left[\int_{0}^{t} \sum_{\chi=0}^{\infty} \frac{\lambda^{\chi}}{\Gamma(\chi \alpha+\alpha)} \int_{u}^{t}(t-s)^{n-\alpha-1}(s-u)^{\alpha-1+\chi \alpha} d s h(u) d u\right]^{(n)}}{\Gamma(n-\alpha)} \\
& +\frac{\left[\sum_{k=0}^{\omega} \sum_{\nu=1}^{n} c_{\nu k} \sum_{\chi=0}^{\infty} \frac{\lambda^{\chi}}{\Gamma(\chi \alpha+\alpha-\nu+1)} \int_{t_{k}}^{t}(t-s)^{n-\alpha-1}\left(s-t_{k}\right)^{\alpha-\nu+\chi \alpha} d s\right]^{(n)}}{\Gamma(n-\alpha)}
\end{aligned}
$$

by

$$
\begin{gathered}
\frac{s-u}{t-u}=w, \frac{s-t_{k}}{t-t_{k}}=w \\
=D_{t_{\omega+1}^{+}}^{\alpha} \Phi(t)+\frac{\left[\int_{0}^{t} \sum_{\chi=0}^{\infty} \frac{\lambda^{\chi}}{\Gamma(\chi \alpha+\alpha)}(t-u)^{\chi \alpha+n-1} \int_{0}^{1}(1-w)^{n-\alpha-1} w^{\alpha-1+\chi \alpha} d w h(u) d u\right]^{(n)}}{\Gamma(n-\alpha)} \\
+\frac{\left[\sum_{k=0}^{\omega} \sum_{\nu=1}^{n} c_{\nu k} \sum_{\chi=0}^{\infty} \frac{\lambda^{\chi}}{\Gamma(\chi \alpha+\alpha-\nu+1)}\left(t-t_{k}\right)^{\chi \alpha+n-\nu} \int_{0}^{1}(1-w)^{n-\alpha-1} w^{\alpha-\nu+\chi \alpha} d w\right]^{(n)}}{\Gamma(n-\alpha)} \\
=D_{t_{\omega+1}^{+}}^{\alpha} \Phi(t)+h(t)+\left[\int_{0}^{t} \frac{\left.\sum_{\chi=0}^{\infty} \frac{\lambda^{\chi}(t-u)^{\chi \alpha+n-1}}{\Gamma(\chi \alpha+n)} h(u) d u\right]^{(n)}}{\left.+\sum_{k=0}^{\omega} \sum_{\nu=1}^{n} c_{\nu k} \sum_{\chi=0}^{\infty} \frac{\lambda^{\chi}\left(t-t_{k}\right)^{\chi \alpha+n-\nu}}{\Gamma(\chi \alpha+n-\nu+1)}\right]^{(n)}}\right. \\
-D_{t_{\omega+1}^{+}}^{\alpha}(t)+\int_{0}^{t} \sum_{\chi=1}^{\infty} \frac{\lambda^{\chi}(t-u)^{\chi \alpha+-1}}{\Gamma(\chi \alpha)} h(u) d u+\sum_{k=0}^{\omega} \sum_{\nu=1}^{n} c_{\nu k} \sum_{\chi=1}^{\infty} \frac{\lambda^{\chi}\left(t-t_{k}\right)^{\chi \alpha-\nu}}{\Gamma(\chi \alpha-\nu+1)}
\end{gathered}
$$

Thus

$$
\begin{aligned}
D_{0^{+}}^{\alpha} x(t)-\lambda x(t)= & D_{t_{\omega+1}^{+}}^{\alpha} \Phi(t)+h(t)+\int_{0}^{t} \sum_{\chi=1}^{\infty} \frac{\lambda^{\chi}(t-u)^{\chi \alpha+-1}}{\Gamma(\chi \alpha)} h(u) d u \\
& +\sum_{k=0}^{\omega} \sum_{\nu=1}^{n} c_{\nu k} \sum_{\chi=1}^{\infty} \frac{\lambda^{\chi}\left(t-t_{k}\right)^{\chi \alpha-\nu}}{\Gamma(\chi \alpha-\nu+1)} \\
& -\lambda\left[\Phi(t)+\int_{0}^{t}(t-s)^{\alpha-1} \mathbf{E}_{\alpha, \alpha}\left(\lambda(t-s)^{\alpha}\right) h(s) d s\right.
\end{aligned}
$$




$$
\left.+\sum_{k=0}^{\omega} \sum_{\nu=1}^{n} c_{\nu k}\left(t-t_{k}\right)^{\alpha-\nu} \mathbf{E}_{\alpha, \alpha-\nu+1}\left(\lambda\left(t-t_{k}\right)^{\alpha}\right)\right]=D_{t_{\omega+1}^{+}}^{\alpha} \Phi(t)-\lambda \Phi(t)+h(t) .
$$

From $D_{0^{+}}^{\alpha} x(t)-\lambda x(t)=h(t)$, we have $D_{t_{\omega+1}^{+}}^{\alpha} \Phi(t)-\lambda \Phi(t)=0$ on $\left(t_{\omega+1}, t_{\omega+2}\right]$.

By (5.1)-(5.3) in [7], we know that there exists constants $c_{\nu \omega+1} \in \mathbb{R}$ such that

$$
\Phi(t)=\sum_{\nu=1}^{n} c_{\nu \omega+1}\left(t-t_{\omega+1}\right)^{\alpha-\nu} \mathbf{E}_{\alpha, \alpha}\left(\lambda\left(t-t_{\omega+1}\right)^{\alpha}\right) .
$$

Substituting $\Phi$ into (2.4). We know that (2.2) holds for $i=\omega+1$. By mathematical induction method, we know that (2.2) holds for $i \in \mathbb{N}_{0}^{m}$.

Step 2. We prove that $x$ is a piecewise continuous solution of (2.1) if $x$ satisfies (2.2). Since $x$ satisfies $(2.2)$, we know that $\left.x\right|_{\left(t_{i}, t_{i+1}\right]} \in C^{0}\left(t_{i}, t_{i+1}\right]\left(i \in \mathbb{N}_{0}^{m}\right)$ and

$$
\lim _{t \rightarrow t_{j}^{+}}\left(t-t_{j}\right)^{n-\alpha} x(t)
$$

exists and is finite for all $i \in \mathbb{N}_{0}^{m}$. Furthermore, by direct computation similarly to Step 1 , by Definition 2.1, we can get for $t \in\left(t_{j}, t_{j+1}\right]$ that

$$
\begin{gathered}
I_{0^{+}}^{n-\alpha} x(t)=\frac{\int_{0}^{t}(t-s)^{n-\alpha-1} x(s) d s}{\Gamma(\alpha)} \\
=\frac{\sum_{\rho=0}^{j-1} \int_{t_{\rho}}^{t_{\rho}+1}(t-s)^{n-\alpha-1} x(s) d s+\int_{t_{j}}^{t}(t-s)^{n-\alpha-1} x(s) d s}{\Gamma(n-\alpha)} \\
=\int_{0}^{t} \sum_{\chi=0}^{\infty} \frac{\lambda^{\chi}}{\Gamma(\chi \alpha+n)}(t-u)^{\chi \alpha+n-1} h(u) d u \\
+\sum_{k=0}^{j} \sum_{\nu=1}^{n} c_{\nu k} \sum_{\chi=0}^{\infty} \frac{\lambda}{\Gamma(\chi \alpha+n-\nu+1)}\left(t-t_{k}\right)^{\chi \alpha+n-\nu} \\
=\int_{0}^{t}(t-u)^{n-1} \mathbf{E}_{\alpha, n}\left(\lambda(t-s)^{\alpha}\right) h(s) d s+\sum_{k=0}^{j} \sum_{\nu=1}^{n} c_{\nu k}\left(t-t_{k}\right)^{n-\nu} \mathbf{E}_{\alpha, n-\nu+1}\left(\lambda\left(t-t_{k}\right)^{\alpha}\right) .
\end{gathered}
$$

By Definition 2.2, we get for $i \in \mathbb{N}_{1}^{n-1}, t \in\left(t_{j} t_{j+1}\right]$ that

$$
\begin{gathered}
D_{0^{+}}^{\alpha-i} x(t)=\frac{\left[\int_{0}^{t}(t-s)^{n-\alpha-1} x(s) d s\right]^{(n-i)}}{\Gamma(\alpha)} \\
=\frac{\left[\sum_{\rho=0}^{j-1} \int_{t_{\rho}}^{t_{\rho}+1}(t-s)^{n-\alpha-1} x(s) d s+\int_{t_{j}}^{t}(t-s)^{n-\alpha-1} x(s) d s\right]^{(n-i)}}{\Gamma(n-\alpha)} \\
=\left[\int_{0}^{t} \sum_{\chi=0}^{\infty} \frac{\lambda^{\chi}(t-u)^{\chi \alpha+n-1}}{\Gamma(\chi \alpha+n)} h(u) d u\right]^{(n-i)}+\left[\sum_{k=0}^{j} \sum_{\nu=1}^{n} c_{\nu k} \sum_{\chi=0}^{\infty} \frac{\lambda^{\chi}\left(t-t_{k}\right)^{\chi \alpha+n-\nu}}{\Gamma(\chi \alpha+n-\nu+1)}\right]^{(n-i)}
\end{gathered}
$$




$$
\begin{gathered}
=\int_{0}^{t} \sum_{\chi=0}^{\infty} \frac{\lambda^{\chi}(t-u)^{\chi \alpha+i-1}}{\Gamma(\chi \alpha+i)} h(u) d u \\
+\sum_{k=0}^{j} \sum_{\nu=1}^{n} c_{\nu k} \sum_{\chi=1}^{\infty} \frac{\lambda^{\chi}\left(t-t_{k}\right)^{\chi \alpha+i-\nu}}{\Gamma(\chi \alpha+i-\nu+1)}+\sum_{k=0}^{j} \sum_{\nu=1}^{i} c_{\nu k} \frac{\left(t-t_{k}\right)^{i-\nu}}{\Gamma(i-\nu+1)} \\
=\int_{0}^{t}(t-u)^{i-1} \mathbf{E}_{\alpha, i}\left(\lambda(t-u)^{\alpha}\right) h(u) d u+\sum_{k=0}^{j} \sum_{\nu=1}^{i} c_{\nu k}\left(t-t_{k}\right)^{i-\nu} \mathbf{E}_{\alpha, i-\nu+1}\left(\lambda\left(t-t_{k}\right)^{\alpha}\right) \\
+\lambda \sum_{k=0}^{j} \sum_{\nu=i+1}^{n} c_{\nu k}\left(t-t_{k}\right)^{\alpha+i-\nu} \mathbf{E}_{\alpha, \alpha+i-\nu+1}\left(\lambda\left(t-t_{k}\right)^{\alpha}\right) .
\end{gathered}
$$

We see that

$$
\left.I_{0^{+}}^{n-\alpha} x\right|_{\left(t_{j}, t_{j+1}\right]},\left.D_{0^{+}}^{\alpha-i} x\right|_{\left(t_{j}, t_{j+1}\right]}\left(i \in \mathbb{N}_{1}^{n-1}, j \in \mathbb{N}_{0}^{m}\right)
$$

are continuous and the limits

$$
\lim _{t \rightarrow t_{j}^{+}} I_{0^{+}}^{n-\alpha} x(t), \quad \lim _{t \rightarrow t_{j}^{+}} D_{0^{+}}^{\alpha-i} x(t) \quad\left(i \in \mathbb{N}_{1}^{n-1}, j \in \mathbb{N}_{0}^{m}\right)
$$

are finite. By Definition 2.2, $\alpha \in(n-1, n)$, for $t \in\left(t_{j}, t_{j+1}\right]$, we have

$$
\begin{gathered}
D_{0^{+}}^{\alpha} x(t)=\frac{\left[\int_{0}^{t}(t-s)^{n-\alpha-1} x(s) d s\right]^{(n-i)}}{\Gamma(\alpha)} \\
\left.=\frac{\left[\sum_{\rho=0}^{j-1} \int_{t_{\rho}+1}^{t_{\rho}+1}(t-s)^{n-\alpha-1} x(s) d s+\int_{t_{j}}^{t}(t-s)^{n-\alpha-1} x(s) d s\right]^{(n-i)}}{\Gamma(n-\alpha)}\right]^{(n)}+\left[\sum_{k=0}^{j} \sum_{\nu=1}^{n} c_{\nu k} \sum_{\chi=0}^{\infty} \frac{\lambda^{\chi}\left(t-t_{k}\right)^{\chi \alpha+n-\nu}}{\Gamma(\chi \alpha+n-\nu+1)}\right]^{(n)} \\
=\left[\int_{0}^{t} \sum_{\chi=0}^{\infty} \frac{\lambda^{\chi}(t-u)^{\chi \alpha+n-1}}{\Gamma(\chi \alpha+n)} h(u) d u\right. \\
=h(t)+\int_{0}^{t} \sum_{\chi=1}^{\infty} \frac{\lambda^{\chi}(t-u)^{\chi \alpha-1}}{\Gamma(\chi \alpha)} h(u) d u+\sum_{k=0}^{j} \sum_{\nu=1}^{n} c_{\nu k} \sum_{\chi=1}^{\infty} \frac{\lambda^{\chi}\left(t-t_{k}\right)^{\chi \alpha+-\nu}}{\Gamma(\chi \alpha-\nu+1)}, t \in\left(t_{j}, t_{j+1}\right] .
\end{gathered}
$$

Then

$$
D_{0^{+}}^{\alpha} x(t)-\lambda x(t)=h(t), \quad t \in\left(t_{j}, t_{j+1}\right], j \in \mathbb{N}_{0}^{m} .
$$

So $x$ is a piecewise continuous solution of (2.1). The proof is completed.

Remark 2.1. Lemma 2.1 (when $\lambda=0$ ) is one of the main results in [10] (see Theorem 3.2 in [10]). So our results generalizes the one in [10].

Theorem 2.2. Suppose that $x$ is a solution of (2.1) and is defined by (2.2). Then

$$
\begin{aligned}
I_{0^{+}}^{\beta} x(t) & =\int_{0}^{t}(t-s)^{\alpha+\beta-1} \mathbf{E}_{\alpha, \alpha+\beta}\left(\lambda(t-s)^{\alpha}\right) h(s) d s \\
& +\sum_{k=0}^{j} \sum_{\nu=1}^{n} c_{\nu k}\left(t-t_{k}\right)^{\alpha+\beta-\nu} \mathbf{E}_{\alpha, \alpha+\beta-\nu+1}\left(\lambda\left(t-t_{k}\right)^{\alpha}\right), \\
& t \in\left(t_{j}, t_{j+1}\right], j \in \mathbb{N}_{0}^{m}
\end{aligned}
$$


Solvability of BVPs for impulsive fractional differential equations

$$
\begin{aligned}
I_{0^{+}}^{n-\alpha} x(t) & =\sum_{k=0}^{i} \sum_{\nu=1}^{n} c_{\nu k}\left(t-t_{k}\right)^{n-\nu} \mathbf{E}_{\alpha, n-\nu+1}\left(\lambda\left(t-t_{k}\right)^{\alpha}\right) \\
& +\int_{0}^{t}(t-u)^{n-1} \mathbf{E}_{\alpha, n}\left(\lambda(t-u)^{\alpha}\right) h(u) d u \\
& t \in\left(t_{i}, t_{i+1}\right], i \in \mathbb{N}_{0}^{m},
\end{aligned}
$$

and

$$
\begin{aligned}
D_{0^{+}}^{\alpha-j} x(t) & =\sum_{k=0}^{i} \sum_{\nu=1}^{j} c_{\nu k}\left(t-t_{k}\right)^{j-\nu} \mathbf{E}_{\alpha, j-\nu+1}\left(\lambda\left(t-t_{k}\right)^{\alpha}\right) \\
& +\lambda \sum_{k=0}^{i} \sum_{\nu=j+1}^{n} c_{\nu k}\left(t-t_{k}\right)^{\alpha+j-\nu} \mathbf{E}_{\alpha, \alpha+j-\nu+1}\left(\lambda\left(t-t_{k}\right)^{\alpha}\right) \\
& +\int_{0}^{t}(t-u)^{j-1} \mathbf{E}_{\alpha, j}\left(\lambda(t-u)^{\alpha}\right) h(u) d u, \\
& t \in\left(t_{i}, t_{i+1}\right], i \in \mathbb{N}_{0}^{m}, j \in \mathbb{N}_{1}^{n-1} .
\end{aligned}
$$

Proof. We firstly prove (2.5). For $t \in\left(t_{i}, t_{i+1}\right]$, by (2.2) and Definition 2.1, we get

$$
\begin{aligned}
& I_{0^{+}}^{\beta} x(t)=\frac{\sum_{\mu=0}^{i-1} \int_{t_{\mu}}^{t_{\mu+1}}(t-s)^{\beta-1} x(s) d s+\int_{t_{i}}^{t}(t-s)^{\beta-1} x(s) d s}{\Gamma(\beta)} \\
& =\sum_{\mu=0}^{i-1} \int_{t_{\mu}}^{t_{\mu+1}}(t-s)^{\beta-1}\left(\int_{0}^{s}(s-u)^{\alpha-1} \mathbf{E}_{\alpha, \alpha}\left(\lambda(s-u)^{\alpha}\right) h(u) d u\right. \\
& \left.+\sum_{k=0}^{\mu} \sum_{\nu=1}^{n} c_{\nu k}\left(s-t_{k}\right)^{\alpha-\nu} \mathbf{E}_{\alpha, \alpha-\nu+1}\left(\lambda\left(s-t_{k}\right)^{\alpha}\right)\right) d s / \Gamma(\beta) \\
& +\int_{t_{i}}^{t}(t-s)^{\beta-1}\left(\int_{0}^{s}(s-u)^{\alpha-1} \mathbf{E}_{\alpha, \alpha}\left(\lambda(s-u)^{\alpha}\right) h(u) d u\right. \\
& \left.+\sum_{k=0}^{i} \sum_{\nu=1}^{n} c_{\nu k}\left(s-t_{k}\right)^{\alpha-\nu} \mathbf{E}_{\alpha, \alpha-\nu+1}\left(\lambda\left(s-t_{k}\right)^{\alpha}\right)\right) d s / \Gamma(\beta) \\
& =\frac{\sum_{\mu=0}^{i-1} \int_{t_{\mu}}^{t_{\mu+1}}(t-s)^{\beta-1} \sum_{k=0}^{\mu} \sum_{\nu=1}^{n} c_{\nu k}\left(s-t_{k}\right)^{\alpha-\nu} \mathbf{E}_{\alpha, \alpha-\nu+1}\left(\lambda\left(s-t_{k}\right)^{\alpha}\right) d s}{\Gamma(\beta)} \\
& +\frac{\int_{t_{i}}^{t}(t-s)^{\beta-1} \sum_{k=0}^{i} \sum_{\nu=1}^{n} c_{\nu k}\left(s-t_{k}\right)^{\alpha-\nu} \mathbf{E}_{\alpha, \alpha-\nu+1}\left(\lambda\left(s-t_{k}\right)^{\alpha}\right) d s}{\Gamma(\beta)} \\
& +\frac{\int_{0}^{t}(t-s)^{\beta-1} \int_{0}^{s}(s-u)^{\alpha-1} \mathbf{E}_{\alpha, \alpha}\left(\lambda(s-u)^{\alpha}\right) h(u) d u d s}{\Gamma(\beta)}
\end{aligned}
$$




$$
\begin{aligned}
= & \frac{\sum_{k=0}^{i-1} \sum_{\mu=k}^{i-1} \sum_{\nu=1}^{n} c_{\nu k} \int_{t_{\mu}}^{t_{\mu+1}}(t-s)^{\beta-1}\left(s-t_{k}\right)^{\alpha-\nu} \sum_{\chi=0}^{\infty} \frac{\lambda^{\chi}}{\Gamma\left(\chi^{\alpha+\alpha}-\nu+1\right)}\left(s-t_{k}\right)^{\chi \alpha} d s}{\Gamma(\beta)} \\
+ & \frac{\sum_{k=0}^{i} \sum_{\nu=1}^{n} c_{\nu k} \int_{t_{i}}^{t}(t-s)^{\beta-1}\left(s-t_{k}\right)^{\alpha-\nu} \sum_{\chi=0}^{\infty} \frac{\lambda^{\chi}}{\Gamma\left(\chi^{\alpha+\alpha-\nu+1)}\right.}\left(s-t_{k}\right)^{\chi \alpha} d s}{\Gamma(\beta)} \\
& +\frac{\int_{0}^{t} \int_{u}^{t}(t-s)^{\beta-1}(s-u)^{\alpha-1} \sum_{\chi=0}^{\infty} \frac{\lambda^{\chi}}{\Gamma(\chi \alpha+\alpha)}(s-u)^{\chi \alpha} d s h(u) d u}{\Gamma(\beta)} \\
& \sum_{k=0}^{i-1} \sum_{\mu=k}^{i-1} \sum_{\nu=1}^{n} c_{\nu k} \sum_{\chi=0}^{\infty} \frac{\lambda^{\chi}}{\Gamma(\chi \alpha+\alpha-\nu+1)} \int_{t_{\mu}}^{t_{\mu+1}}(t-s)^{\beta-1}\left(s-t_{k}\right)^{\alpha-\nu+\chi \alpha} d s \\
& +\frac{\Gamma(\beta)}{\sum_{k=0}^{i} \sum_{\nu=1}^{n} c_{\nu k} \sum_{\chi=0}^{\infty} \frac{\lambda^{\chi}}{\Gamma(\chi \alpha+\alpha-\nu+1)} \int_{t_{i}}^{t}(t-s)^{\beta-1}\left(s-t_{k}\right)^{\alpha-\nu+\chi \alpha} d s} \\
& +\frac{\sum_{\chi=0}^{\infty} \frac{\lambda^{\chi}}{\Gamma(\chi \alpha+\alpha)} \int_{0}^{t} \int_{u}^{t}(t-s)^{\beta-1}(s-u)^{\alpha-1+\chi \alpha} d s h(u) d u}{\Gamma(\beta)}
\end{aligned}
$$

$=\frac{\left.\sum_{k=0}^{i-1} \sum_{\mu=k}^{i-1} \sum_{\nu=1}^{n} c_{\nu k} \sum_{\chi=0}^{\infty} \frac{\lambda^{\chi}}{\Gamma\left(\chi^{\alpha+\alpha-\nu+1)}\right.} t-t_{k}\right)^{\chi \alpha+\alpha+\beta-\nu} \int_{\frac{t_{\mu}-t_{K}}{t-t_{k}}}^{\frac{t_{\mu+1}-t_{k}}{t-t_{k}}}(1-w)^{\beta-1} w^{\alpha-\nu+\chi \alpha} d w}{\Gamma(\beta)}$ $+\frac{\sum_{k=0}^{i} \sum_{\nu=1}^{n} c_{\nu k} \sum_{\chi=0}^{\infty} \frac{\lambda^{\chi}}{\Gamma(\chi \alpha+\alpha-\nu+1)}\left(t-t_{k}\right)^{\chi \alpha+\alpha+\beta-\nu} \int_{\frac{t_{i}-t_{k}}{t-t_{k}}}^{1}(1-w)^{\beta-1} w^{\alpha-\nu+\chi \alpha} d w}{\Gamma(\beta)}$

$$
\begin{gathered}
+\frac{\sum_{\chi=0}^{\infty} \frac{\lambda^{\chi}}{\Gamma\left(\chi^{\alpha+\alpha}\right)} \int_{0}^{t}(t-u)^{\chi \alpha+\alpha+\beta-1} \int_{0}^{1}(1-w)^{\beta-1} w^{\alpha-1+\chi \alpha} d w h(u) d u}{\Gamma(\beta)} \\
=\frac{\sum_{k=0}^{i} \sum_{\nu=1}^{n} c_{\nu k} \sum_{\chi=0}^{\infty} \frac{\lambda^{\chi}}{\Gamma\left(\chi^{\alpha+\alpha-\nu+1)}\right.}\left(t-t_{k}\right)^{\chi \alpha+\alpha+\beta-\nu} \int_{0}^{1}(1-w)^{\beta-1} w^{\alpha-\nu+\chi \alpha} d w}{\Gamma(\beta)} \\
+\frac{\sum_{\chi=0}^{\infty} \frac{\lambda^{\chi}}{\Gamma\left(\chi^{\alpha+\alpha)}\right.} \int_{0}^{t}(t-u)^{\chi \alpha+\alpha+\beta-1} \int_{0}^{1}(1-w)^{\beta-1} w^{\alpha-1+\chi \alpha} d w h(u) d u}{\Gamma(\beta)} \\
=\sum_{k=0}^{i} \sum_{\nu=1}^{n} c_{\nu k} \sum_{\chi=0}^{\infty} \frac{\lambda^{\chi}}{\Gamma(\chi \alpha+\alpha+\beta-\nu+1)}\left(t-t_{k}\right)^{\chi \alpha+\alpha+\beta-\nu} \\
+\sum_{\chi=0}^{\infty} \frac{\lambda^{\chi}}{\Gamma(\chi \alpha+\alpha+\beta)} \int_{0}^{t}(t-u)^{\chi \alpha+\alpha+\beta-1} h(u) d u
\end{gathered}
$$


Solvability of BVPs for impulsive fractional differential equations

$$
\begin{gathered}
=\int_{0}^{t}(t-s)^{\alpha+\beta-1} \mathbf{E}_{\alpha, \alpha+\beta}\left(\lambda(t-s)^{\alpha}\right) h(s) d s \\
+\sum_{k=0}^{i} \sum_{\nu=1}^{n} c_{\nu k}\left(t-t_{k}\right)^{\alpha+\beta-\nu} \mathbf{E}_{\alpha, \alpha+\beta-\nu+1}\left(\lambda\left(t-t_{k}\right)^{\alpha}\right), t \in\left(t_{i}, t_{i+1}\right], i \in \mathbb{N}_{0}^{m}
\end{gathered}
$$

Thus (2.5) is proved. Hence (2.6) holds by $\beta=n-\alpha$.

Now, we prove (2.7). In fact, for $t \in\left(t_{i}, t_{i+1}\right]$, we have by using 2.2 and Definition 2.2 that

$$
\begin{aligned}
& D_{0^{+}}^{\alpha-j} x(t)=\frac{\left[\int_{0}^{t}(t-s)^{n-\alpha-1} x(s) d s\right]^{(n-j)}}{\Gamma(n-\alpha)} \\
& =\frac{\left[\sum_{\mu=0}^{i-1}(t-s)^{n-\alpha-1} x(s) d s+\int_{t_{i}}^{t}(t-s)^{n-\alpha-1} x(s) d s\right]^{(n-j)}}{\Gamma(n-\alpha)} \\
& =\left[\sum _ { \mu = 0 } ^ { i - 1 } \int _ { t _ { \mu } } ^ { t _ { \mu + 1 } } ( t - s ) ^ { n - \alpha - 1 } \left(\int_{0}^{s}(s-u)^{\alpha-1} \mathbf{E}_{\alpha, \alpha}\left(\lambda(s-u)^{\alpha}\right) h(u) d u\right.\right. \\
& \left.\left.+\sum_{k=0}^{\mu} \sum_{\nu=1}^{n} c_{\nu k}\left(s-t_{k}\right)^{\alpha-\nu} \mathbf{E}_{\alpha, \alpha-\nu+1}\left(\lambda\left(s-t_{k}\right)^{\alpha}\right)\right) d s\right]^{(n-j)} / \Gamma(n-\alpha) \\
& +\left[\int _ { t _ { i } } ^ { t } ( t - s ) ^ { n - \alpha - 1 } \left(\int_{0}^{s}(s-u)^{\alpha-1} \mathbf{E}_{\alpha, \alpha}\left(\lambda(s-u)^{\alpha}\right) h(u) d u\right.\right. \\
& \left.\left.+\sum_{k=0}^{i} \sum_{\nu=1}^{n} c_{\nu k}\left(s-t_{k}\right)^{\alpha-\nu} \mathbf{E}_{\alpha, \alpha-\nu+1}\left(\lambda\left(s-t_{k}\right)^{\alpha}\right)\right) d s\right]^{(n-j)} / \Gamma(n-\alpha) \\
& =\frac{\left[\sum_{\mu=0}^{i-1} \int_{t_{\mu}}^{t_{\mu+1}}(t-s)^{n-\alpha-1} \sum_{k=0}^{\mu} \sum_{\nu=1}^{n} c_{\nu k}\left(s-t_{k}\right)^{\alpha-\nu} \mathbf{E}_{\alpha, \alpha-\nu+1}\left(\lambda\left(s-t_{k}\right)^{\alpha}\right) d s\right]^{(n-j)}}{\Gamma(n-\alpha)} \\
& +\frac{\left[\int_{t_{i}}^{t}(t-s)^{n-\alpha-1} \sum_{k=0}^{i} \sum_{\nu=1}^{n} c_{\nu k}\left(s-t_{k}\right)^{\alpha-\nu} \mathbf{E}_{\alpha, \alpha-\nu+1}\left(\lambda\left(s-t_{k}\right)^{\alpha}\right) d s\right]^{(n-j)}}{\Gamma(n-\alpha)} \\
& +\frac{\left[\int_{0}^{t}(t-s)^{n-\alpha-1} \int_{0}^{s}(s-u)^{\alpha-1} \mathbf{E}_{\alpha, \alpha}\left(\lambda(s-u)^{\alpha}\right) h(u) d u d s\right]^{(n-j)}}{\Gamma(n-\alpha)} \\
& =\frac{\left[\sum_{k=0}^{i-1} \sum_{\mu=k}^{i-1} \sum_{\nu=1}^{n} c_{\nu k} \int_{t_{\mu}}^{t_{\mu+1}}(t-s)^{n-\alpha-1}\left(s-t_{k}\right)^{\alpha-\nu} \sum_{\chi=0}^{\infty} \frac{\lambda^{\chi}}{\Gamma(\chi \alpha+\alpha-\nu+1)}\left(s-t_{k}\right)^{\chi \alpha} d s\right]^{(n-j)}}{\Gamma(n-\alpha)} \\
& +\frac{\left[\sum_{k=0}^{i} \sum_{\nu=1}^{n} c_{\nu k} \int_{t_{i}}^{t}(t-s)^{n-\alpha-1}\left(s-t_{k}\right)^{\alpha-\nu} \sum_{\chi=0}^{\infty} \frac{\lambda^{\chi}}{\Gamma(\chi \alpha+\alpha-\nu+1)}\left(s-t_{k}\right)^{\chi \alpha} d s\right]^{(n-j)}}{\Gamma(n-\alpha)}
\end{aligned}
$$




$$
\begin{aligned}
& +\frac{\left[\int_{0}^{t} \int_{u}^{t}(t-s)^{n-\alpha-1}(s-u)^{\alpha-1} \sum_{\chi=0}^{\infty} \frac{\lambda^{\chi}}{\Gamma\left(\chi^{\alpha+\alpha)}\right.}(s-u)^{\chi \alpha} d s h(u) d u\right]^{(n-j)}}{\Gamma(n-\alpha)} \\
& =\frac{\left[\sum_{k=0}^{i-1} \sum_{\mu=k}^{i-1} \sum_{\nu=1}^{n} c_{\nu k} \sum_{\chi=0}^{\infty} \frac{\lambda^{\chi}}{\Gamma\left(\chi^{\alpha+\alpha-\nu+1)}\right.} \int_{t_{\mu}}^{t_{\mu+1}}(t-s)^{n-\alpha-1}\left(s-t_{k}\right)^{\alpha-\nu+\chi \alpha} d s\right]^{(n-j)}}{\Gamma(n-\alpha)} \\
& +\frac{\left[\sum_{k=0}^{i} \sum_{\nu=1}^{n} c_{\nu k} \sum_{\chi=0}^{\infty} \frac{\lambda^{\chi}}{\Gamma(\chi \alpha+\alpha-\nu+1)} \int_{t_{i}}^{t}(t-s)^{n-\alpha-1}\left(s-t_{k}\right)^{\alpha-\nu+\chi \alpha} d s\right]^{(n-j)}}{\Gamma(n-\alpha)} \\
& +\frac{\left[\sum_{\chi=0}^{\infty} \frac{\lambda^{\chi}}{\Gamma\left(\chi^{\alpha+\alpha}\right)} \int_{0}^{t} \int_{u}^{t}(t-s)^{n-\alpha-1}(s-u)^{\alpha-1+\chi \alpha} d s h(u) d u\right]^{(n-j)}}{\Gamma(n-\alpha)} \\
& =\left[\sum_{k=0}^{i-1} \sum_{\mu=k}^{i-1} \sum_{\nu=1}^{n} c_{\nu k} \sum_{\chi=0}^{\infty} \frac{\lambda^{\chi}}{\Gamma(\chi \alpha+\alpha-\nu+1)}\left(t-t_{k}\right)^{\chi \alpha+n-\nu}\right. \\
& \left.\times \int_{\frac{t_{\mu}-t_{k}}{t-t_{k}}}^{\frac{t_{\mu+1}-t_{k}}{t-t_{k}}}(1-w)^{n-\alpha-1} w^{\alpha-\nu+\chi \alpha} d w\right]^{(n-j)} / \Gamma(n-\alpha) \\
& +\frac{\left[\sum_{k=0}^{i} \sum_{\nu=1}^{n} c_{\nu k} \sum_{\chi=0}^{\infty} \frac{\lambda^{\chi}}{\Gamma(\chi \alpha+\alpha-\nu+1)}\left(t-t_{k}\right)^{\chi \alpha+n-\nu} \int_{\frac{t_{i}-t_{k}}{t-t_{k}}}^{1}(1-w)^{n-\alpha-1} w^{\alpha-\nu+\chi \alpha} d w\right]^{(n-j)}}{\Gamma(n-\alpha)} \\
& +\frac{\left[\sum_{\chi=0}^{\infty} \frac{\lambda^{\chi}}{\Gamma\left(\chi^{\alpha+\alpha)}\right.} \int_{0}^{t}(t-u)^{\chi \alpha+n-1} \int_{0}^{1}(1-w)^{n-\alpha-1} w^{\alpha-1+\chi \alpha} d w h(u) d u\right]^{(n-j)}}{\Gamma(n-\alpha)} \\
& =\frac{\left[\sum_{k=0}^{i} \sum_{\nu=1}^{n} c_{\nu k} \sum_{\chi=0}^{\infty} \frac{\lambda^{\chi}}{\Gamma(\chi \alpha+\alpha-\nu+1)}\left(t-t_{k}\right)^{\chi \alpha+n-\nu} \int_{0}^{1}(1-w)^{n-\alpha-1} w^{\alpha-\nu+\chi \alpha} d w\right]^{(n-j)}}{\Gamma(n-\alpha)} \\
& +\frac{\left[\sum_{\chi=0}^{\infty} \frac{\lambda^{\chi}}{\Gamma\left(\chi^{\alpha+\alpha)}\right.} \int_{0}^{t}(t-u)^{\chi \alpha+n-1} \int_{0}^{1}(1-w)^{n-\alpha-1} w^{\alpha-1+\chi \alpha} d w h(u) d u\right]^{(n-j)}}{\Gamma(n-\alpha)} \\
& =\left[\sum_{k=0}^{i} \sum_{\nu=1}^{n} c_{\nu k} \sum_{\chi=0}^{\infty} \frac{\lambda^{\chi}}{\Gamma(\chi \alpha+n-\nu+1)}\left(t-t_{k}\right)^{\chi \alpha+n-\nu}\right]^{(n-j)}
\end{aligned}
$$


Solvability of BVPs for impulsive fractional differential equations

$$
\begin{aligned}
& +\left[\sum_{\chi=0}^{\infty} \frac{\lambda^{\chi}}{\Gamma(\chi \alpha+n)} \int_{0}^{t}(t-u)^{\chi \alpha+n-1} h(u) d u\right]^{(n-j)} \\
& =\sum_{k=0}^{i} \sum_{\nu=1}^{j} c_{\nu k} \frac{1}{\Gamma(j-\nu+1)}\left(t-t_{k}\right)^{j-\nu}+\sum_{k=0}^{i} \sum_{\nu=1}^{n} c_{\nu k} \sum_{\chi=1}^{\infty} \frac{\lambda^{\chi}}{\Gamma(\chi \alpha+j-\nu+1)}\left(t-t_{k}\right)^{\chi \alpha+j-\nu} \\
& +\sum_{\chi=0}^{\infty} \frac{\lambda^{\chi}}{\Gamma(\chi \alpha+j)} \int_{0}^{t}(t-u)^{\chi \alpha+j-1} h(u) d u \\
& =\sum_{k=0}^{i} \sum_{\nu=1}^{j} c_{\nu k} \sum_{\chi=0}^{\infty} \frac{\lambda^{\chi}}{\Gamma(\chi \alpha+j-\nu+1)}\left(t-t_{k}\right)^{\chi \alpha+j-\nu} \\
& +\sum_{k=0}^{i} \sum_{\nu=j+1}^{n} c_{\nu k} \sum_{\chi=1}^{\infty} \frac{\lambda^{\chi}}{\Gamma(\chi \alpha+j-\nu+1)}\left(t-t_{k}\right)^{\chi \alpha+j-\nu} \\
& +\int_{0}^{t}(t-u)^{j-1} \mathbf{E}_{\alpha, j}\left(\lambda(t-u)^{\alpha}\right) h(u) d u \\
& =\sum_{k=0}^{i} \sum_{\nu=1}^{j} c_{\nu k}\left(t-t_{k}\right)^{j-\nu} \mathbf{E}_{\alpha, j-\nu+1}\left(\lambda\left(t-t_{k}\right)^{\alpha}\right) \\
& +\lambda \sum_{k=0}^{i} \sum_{\nu=j+1}^{n} c_{\nu k}\left(t-t_{k}\right)^{\alpha+j-\nu} \mathbf{E}_{\alpha, \alpha+j-\nu+1}\left(\lambda\left(t-t_{k}\right)^{\alpha}\right) \\
& +\int_{0}^{t}(t-u)^{j-1} \mathbf{E}_{\alpha, j}\left(\lambda(t-u)^{\alpha}\right) h(u) d u .
\end{aligned}
$$

Banach space $P C_{n-\alpha}(0,1]$. Let $n$ be a positive integer, $\alpha \in(n-1, n)$ and $0=t_{0}<$ $t_{1}<\cdots<t_{m+1}=1$. Choose

$$
P C_{n-\alpha}(0,1]=\left\{x:(0,1] \mapsto \mathbf{R}: \begin{array}{c}
\left.x\right|_{\left(t_{i}, t_{i+1}\right]} \in C^{0}\left(t_{i}, t_{i+1}\right], i \in \mathbf{N}_{0}^{m}, \\
\lim _{t \rightarrow t_{i}^{+}}\left(t-t_{i}\right)^{n-\alpha} x(t), i \in \mathbf{N}_{0}^{m} \text { are finite }
\end{array}\right\} .
$$

Define

$$
\|x\|=\|x\|_{n-\alpha}=\max \left\{\sup _{t \in\left(t_{i}, t_{i+1}\right]}\left(t-t_{i}\right)^{n-\alpha}|x(t)|, i \in \mathbf{N}_{0}^{m}\right\} .
$$

Then $P C_{n-\alpha}(0,1]$ is a Banach space with the norm $\|\cdot\|$ defined. 
Theorem 2.3. Suppose that $\alpha+\beta=n, h \in L^{1}(0,1] a_{j i}, b_{i} \in \mathbb{R}$. Then $x \in P C_{n-\alpha}(0,1]$ is a solution of

$$
\left(\begin{array}{c}
D_{0^{+}}^{\alpha} x(t)-\lambda x(t), \text { a.e., } t \in\left(t_{i}, t_{i+1}\right], i \in \mathbb{N}_{0}^{m} \\
\Delta I_{0^{+}}^{n-\alpha} x\left(t_{i}\right), i \in \mathbb{N}_{1}^{m} \\
\Delta D_{0^{+}}^{\alpha-j} x\left(t_{i}\right), i \in \mathbb{N}_{1}^{m}, j \in \mathbb{N}_{1}^{n-1} \\
I_{0^{+}}^{n-\alpha} x(0) \\
D_{0^{+}}^{\alpha-j} x(0), j \in \mathbb{N}_{1}^{n-1}
\end{array}\right)=\left(\begin{array}{c}
h(t), \text { a.e. }, t \in\left(t_{i}, t_{i+1}\right], i \in \mathbb{N}_{0}^{m} \\
a_{n i}, i \in \mathbb{N}_{1}^{m} \\
a_{j i}, i \in \mathbb{N}_{1}^{m}, j \in \mathbb{N}_{1}^{n-1} \\
b_{n} \\
b_{j}, j \in \mathbb{N}_{1}^{n-1}
\end{array}\right)
$$

if and only if

$$
\begin{aligned}
x(t) & =\int_{0}^{t}(t-s)^{\alpha-1} \mathbf{E}_{\alpha, \alpha}\left(\lambda(t-s)^{\alpha}\right) h(s) d s+\sum_{\nu=1}^{n} b_{\nu} t^{\alpha-\nu} \mathbf{E}_{\alpha, \alpha-\nu+1}\left(\lambda t^{\alpha}\right) \\
& +\sum_{k=1}^{j} \sum_{\nu=1}^{n} a_{\nu k}\left(t-t_{k}\right)^{\alpha-\nu} \mathbf{E}_{\alpha, \alpha-\nu+1}\left(\lambda\left(t-t_{k}\right)^{\alpha}\right), t \in\left(t_{j}, t_{j+1}\right], j \in \mathbb{N}_{0}^{m} .
\end{aligned}
$$

Proof. Suppose that $x$ is a solution of (2.15). By Theorem 2.1, there exist constants $c_{\nu k} \in \mathbb{R}\left(\nu \in \mathbb{N}_{1}^{n}, k \in \mathbb{N}_{0}\right)$ such that

$$
\begin{aligned}
x(t) & =\int_{0}^{t}(t-s)^{\alpha-1} \mathbf{E}_{\alpha, \alpha}\left(\lambda(t-s)^{\alpha}\right) h(s) d s \\
& +\sum_{\substack{k=0\\
}} \sum_{\nu=1}^{n} c_{\nu k}\left(t-t_{k}\right)^{\alpha-\nu} \mathbf{E}_{\alpha, \alpha-\nu+1}\left(\lambda\left(t-t_{k}\right)^{\alpha}\right), \\
& t \in\left(t_{j}, t_{j+1}\right], j \in \mathbb{N}_{0}^{m} .
\end{aligned}
$$

Then Theorem 2.2 implies

$$
\begin{aligned}
I_{0^{+}}^{\beta} x(t) & =\int_{0}^{t}(t-s)^{\alpha+\beta-1} \mathbf{E}_{\alpha, \alpha+\beta}\left(\lambda(t-s)^{\alpha}\right) h(s) d s \\
+ & \sum_{k=0}^{j} \sum_{\nu=1}^{n} c_{\nu k}\left(t-t_{k}\right)^{\alpha+\beta-\nu} \mathbf{E}_{\alpha, \alpha+\beta-\nu+1}\left(\lambda\left(t-t_{k}\right)^{\alpha}\right), \\
t & \left(t_{j}, t_{j+1}\right], j \in \mathbb{N}_{0}^{m} \\
I_{0^{+}}^{n-\alpha} x(t) & =\sum_{k=0}^{i} \sum_{\nu=1}^{n} c_{\nu k}\left(t-t_{k}\right)^{n-\nu} \mathbf{E}_{\alpha, n-\nu+1}\left(\lambda\left(t-t_{k}\right)^{\alpha}\right) \\
& +\int_{0}^{t}(t-u)^{n-1} \mathbf{E}_{\alpha, n}\left(\lambda(t-u)^{\alpha}\right) h(u) d u \\
& t \in\left(t_{i}, t_{i+1}\right], i \in \mathbb{N}_{0}^{m}
\end{aligned}
$$


and

$$
\begin{aligned}
D_{0^{+}}^{\alpha-j} x(t) & =\sum_{k=0}^{i} \sum_{\nu=1}^{j} c_{\nu k}\left(t-t_{k}\right)^{j-\nu} \mathbf{E}_{\alpha, j-\nu+1}\left(\lambda\left(t-t_{k}\right)^{\alpha}\right) \\
& +\lambda \sum_{k=0}^{i} \sum_{\nu=j+1}^{n} c_{\nu k}\left(t-t_{k}\right)^{\alpha+j-\nu} \mathbf{E}_{\alpha, \alpha+j-\nu+1}\left(\lambda\left(t-t_{k}\right)^{\alpha}\right) \\
& +\int_{0}^{t}(t-u)^{j-1} \mathbf{E}_{\alpha, j}\left(\lambda(t-u)^{\alpha}\right) h(u) d u, \\
& t \in\left(t_{i}, t_{i+1}\right], i \in \mathbb{N}_{0}^{m}, j \in \mathbb{N}_{1}^{n-1} .
\end{aligned}
$$

By $D_{0^{+}}^{\alpha-j} x(0)=b_{j}$ and $(2.12)$, we get $c_{j 0}=b_{j}, j \in \mathbb{N}_{1}^{n-1}$.

By $I_{0^{+}}^{n-\alpha} x(0)=b_{n}$ and (2.11), we get $c_{n 0}=b_{n}$.

By $\Delta D_{0^{+}}^{\alpha-j} x\left(t_{i}\right)=a_{j i}, i \in \mathbb{N}_{1}^{m}, j \in \mathbb{N}_{1}^{n-1}$ and (2.12), we get $c_{j i}=a_{j i}$ for $i \in \mathbb{N}_{1}^{m}$, $j \in \mathbb{N}_{1}^{n-1}$.

By $\Delta I_{0^{+}}^{n-\alpha} x\left(t_{i}\right)=a_{n i}, i \in \mathbb{N}_{1}^{m}$ and $(2.11)$, we get $c_{n i}=a_{n i}, i \in \mathbb{N}_{1}^{m}$.

Hence

$$
\begin{aligned}
x(t) & =\int_{0}^{t}(t-s)^{\alpha-1} \mathbf{E}_{\alpha, \alpha}\left(\lambda(t-s)^{\alpha}\right) h(s) d s+\sum_{\nu=1}^{n} b_{\nu} t^{\alpha-\nu} \mathbf{E}_{\alpha, \alpha-\nu+1}\left(\lambda t^{\alpha}\right) \\
& +\sum_{k=1}^{j} \sum_{\nu=1}^{n} a_{\nu k}\left(t-t_{k}\right)^{\alpha-\nu} \mathbf{E}_{\alpha, \alpha-\nu+1}\left(\lambda\left(t-t_{k}\right)^{\alpha}\right), t \in\left(t_{j}, t_{j+1}\right], j \in \mathbb{N}_{0}^{m} .
\end{aligned}
$$

The proof is completed.

Theorem 2.4. Suppose that

$$
M=: \frac{1}{\Gamma(\alpha-n+2)}+\sum_{\mu=0}^{m} \int_{t_{\mu}}^{t_{\mu+1}} \frac{s^{\alpha-n+1}}{\Gamma(\alpha-n+2)} d H(s) \neq 0, h \in L^{1}(0,1], a_{j i}, b_{i} \in \mathbb{R} .
$$

Then $x \in P C_{n-\alpha}(0,1]$ is a solution of

$$
\left(\begin{array}{c}
D_{0^{+}}^{\alpha} x(t), \text { a.e. }, t \in\left(t_{i}, t_{i+1}\right], i \in \mathbb{N}_{0}^{m} \\
\Delta I_{0^{+}}^{n-\alpha} x\left(t_{i}\right), i \in \mathbb{N}_{1}^{m} \\
\Delta D_{0^{+}}^{\alpha-j} x\left(t_{i}\right), i \in \mathbb{N}_{1}^{m}, j \in \mathbb{N}_{1}^{n-1}, \\
I_{0^{+}}^{n-\alpha} x(0) \\
D_{0^{+}}^{\alpha-j} x(0)=0, j \in \mathbb{N}_{1}^{n-2} \\
D_{0^{+}}^{\alpha-n+1} x(1)-\int_{0}^{1} x(s) d H(s)
\end{array}\right)=\left(\begin{array}{c}
h(t), a . e ., t \in\left(t_{i}, t_{i+1}\right], i \in \mathbb{N}_{0}^{m} \\
a_{n i}, i \in \mathbb{N}_{1}^{m} \\
a_{j i}, i \in \mathbb{N}_{1}^{m}, j \in \mathbb{N}_{1}^{n-1} \\
0 \\
0, j \in \mathbb{N}_{1}^{n-2} \\
0
\end{array}\right)
$$


if and only if

$$
\begin{aligned}
x(t) & =\int_{0}^{t} \frac{(t-s)^{\alpha-1}}{\Gamma(\alpha)} h(s) d s+\frac{1}{M}\left[\int_{0}^{1}\left(\int_{u}^{1} \frac{(s-u)^{\alpha-1}}{\Gamma(\alpha)} d H(s)-\frac{(1-u)^{\alpha-1}}{\Gamma(\alpha)}\right) h(u) d u\right. \\
& \left.+\sum_{k=1}^{m} \sum_{\nu=1}^{n} a_{\nu k}\left(\int_{t_{k}}^{1} \frac{\left(s-t_{k}\right)^{\alpha-\nu}}{\Gamma(\alpha-\nu+1)} d H(s)-\frac{\left(1-t_{k}\right)^{\alpha-\nu}}{\Gamma(\alpha-\nu+1)}\right)\right] \frac{t^{\alpha-n+1}}{\Gamma(\alpha-n+2)} \\
& +\sum_{k=1}^{j} \sum_{\nu=1}^{n} a_{\nu k} \frac{\left(t-t_{k}\right)^{\alpha-\nu}}{\Gamma(\alpha-\nu+1)}, t \in\left(t_{j}, t_{j+1}\right], j \in \mathbb{N}_{0}^{m} .
\end{aligned}
$$

Proof. By using Theorem $2.1(\lambda=0)$, we get the proof similarly to that of Theorem 2.3 and the proof is omitted.

Define the nonlinear operators $T_{1}, T_{2}$ on $P C_{n-\alpha}(0,1]$ by

$$
\begin{gathered}
\left(T_{1} x\right)(t)=\int_{0}^{t}(t-s)^{\alpha-1} \mathbf{E}_{\alpha, \alpha}\left(\lambda(t-s)^{\alpha}\right) f(s, x(s)) d s+\sum_{\nu=1}^{n} x_{\nu} t^{\alpha-\nu} \mathbf{E}_{\alpha, \alpha-\nu+1}\left(\lambda t^{\alpha}\right) \\
+\sum_{\nu=1}^{n} \sum_{k=1}^{j}\left(t-t_{k}\right)^{\alpha-\nu} \mathbf{E}_{\alpha, \alpha-\nu+1}\left(\lambda\left(t-t_{k}\right)^{\alpha}\right) I_{\nu}\left(t_{k}, x\left(t_{k}\right)\right), t \in\left(t_{j}, t_{j+1}\right], j \in \mathbb{N}_{0}^{m}
\end{gathered}
$$

and

$$
\begin{gathered}
\left(T_{2} x\right)(t)=\int_{0}^{t} \frac{(t-s)^{\alpha-1}}{\Gamma(\alpha)} f(s, x(s)) d s \\
+\frac{1}{M}\left[\int_{0}^{1}\left(\int_{u}^{1} \frac{(s-u)^{\alpha-1}}{\Gamma(\alpha)} d H(s)-\frac{(1-u)^{\alpha-1}}{\Gamma(\alpha)}\right) f(u, x(u)) d u\right. \\
\left.+\sum_{\nu=1}^{n} \sum_{k=1}^{m}\left(\int_{t_{k}}^{1} \frac{\left(s-t_{k}\right)^{\alpha-\nu}}{\Gamma(\alpha-\nu+1)} d H(s)-\frac{\left(1-t_{k}\right)^{\alpha-\nu}}{\Gamma(\alpha-\nu+1)}\right) I_{\nu}\left(t_{k}, x\left(t_{k}\right)\right)\right] \frac{t^{\alpha-n+1}}{\Gamma(\alpha-n+2)} \\
+\sum_{\nu=1}^{n} \sum_{k=1}^{j} \frac{\left(t-t_{k}\right)^{\alpha-\nu}}{\Gamma(\alpha-\nu+1)} I_{\nu}\left(t_{k}, x\left(t_{k}\right)\right), t \in\left(t_{j}, t_{j+1}\right], j \in \mathbb{N}_{0}^{m} .
\end{gathered}
$$

Theorem 2.5. Both $T_{1}: P C_{n-\alpha}(0,1] \rightarrow P C_{n-\alpha}(0,1]$ and $T_{2}: P C_{n-\alpha}(0,1] \rightarrow$ $P C_{n-\alpha}(0,1]$ are well defined and are completely continuous and $x$ is a solution of IVP (1.7) if and only if $x$ is a fixed point of $T_{1}, x$ is a solution of $B V P(1.8)$ if and only if $x$ is a fixed point of $T_{2}$.

Proof. The proof is standard and is omitted.

\section{Main results}

In this section, we establish existence results for IVP (1.6) when $\alpha+\beta \geq n$.

Theorem 3.1. Suppose $\alpha+\beta=n$ and there exist constants $\sigma, A, B, C_{i} \geq 0$ and measurable function $\phi \in L^{1}(0,1)$ such that

$$
\begin{aligned}
& \left|f\left(t,\left(t-t_{i}\right)^{\alpha-n} u\right)-\phi(t)\right| \leq A|u|^{\sigma}, t \in\left(t_{i}, t_{i+1}\right], i \in \mathbb{N}_{0}^{m}, u \in \mathbb{R}, \\
& \left|I_{j}\left(t_{i},\left(t_{i}-t_{i-1}\right)^{\alpha-n} u\right)-C_{i}\right| \leq B|u|^{\sigma}, i \in \mathbb{N}_{1}^{m}, u \in \mathbb{R} .
\end{aligned}
$$


Then IVP (1.7) has at least one solution if $\sigma \in[0,1)$ or $\sigma=1$ with

$$
\frac{\mathbf{E}_{\alpha, \alpha}(|\lambda|)}{\alpha}+m \sum_{\nu=1}^{n} \mathbf{E}_{\alpha, \alpha-\nu+1}(|\lambda|)<1
$$

or $\sigma>1$ with

$$
\left[\frac{\mathbf{E}_{\alpha, \alpha}(|\lambda|)}{\alpha}+m \sum_{\nu=1}^{n} \mathbf{E}_{\alpha, \alpha-\nu+1}(|\lambda|)\right]\left(\frac{\sigma}{\sigma-1}\right)^{\sigma}\|\Phi\|^{\sigma-1}<\frac{1}{\sigma-1} .
$$

where

$$
\begin{aligned}
\Phi(t) & =\int_{0}^{t}(t-s)^{\alpha-1} \mathbf{E}_{\alpha, \alpha}\left(\lambda(t-s)^{\alpha}\right) \phi(s) d s+\sum_{\nu=1}^{n} x_{\nu} t^{\alpha-\nu} \mathbf{E}_{\alpha, \alpha-\nu+1}\left(\lambda t^{\alpha}\right) \\
& +\sum_{\nu=1}^{n} \sum_{k=1}^{j}\left(t-t_{k}\right)^{\alpha-\nu} \mathbf{E}_{\alpha, \alpha-\nu+1}\left(\lambda\left(t-t_{k}\right)^{\alpha}\right) C_{\nu}, t \in\left(t_{j}, t_{j+1}\right], j \in \mathbb{N}_{0}^{m} .
\end{aligned}
$$

Proof. By the definition of $\Phi$, we know $\Phi \in P C_{n-\alpha}(0,1]$. For $r>0$, denote

$$
\Omega_{r}=\left\{x \in P C_{n-\alpha}(0,1]:\|x-\Phi\| \leq r\right\} .
$$

We will seek $r>0$ such that $T_{1} \Omega_{r} \subseteq \Omega_{r}$. Then Schauder's fixed point theorem implies that $T_{1}$ has a fixed point in $\Omega_{r}$. Thus IVP (1.7) has a solution by Theorem 2.5.

For $x \in \Omega_{r}$, we have $\|x\| \leq r+\|\Phi\|$ and

$$
\begin{gathered}
|f(t, x(t))-\phi(t)|=\left|f\left(t,\left(t-t_{i}\right)^{\alpha-n}\left(t-t_{i}\right)^{n-\alpha} x(t)\right)-\phi(t)\right| \\
\left.\leq A \mid\left(t-t_{i}\right)^{n-\alpha} x(t)\right)\left.\right|^{\sigma} \leq A\|x\|^{\sigma} \leq A[r+\|\Phi\|]^{\sigma}, t \in\left(t_{i}, t_{i+1}\right], i \in \mathbb{N}_{0}^{m}, \\
\left|I_{j}\left(t_{i}, x\left(t_{i}\right)\right)-C_{i}\right| \leq B\|x\|^{\sigma} \leq B[r+\|\Phi\|]^{\sigma}, i \in \mathbb{N}_{1}^{m} .
\end{gathered}
$$

For $t \in\left(t_{j}, t_{j+1}\right]$, we have

$$
\begin{gathered}
\left(t-t_{j}\right)^{n-\alpha}\left|\left(T_{1} x\right)(t)-\Phi(t)\right| \\
\leq\left(t-t_{j}\right)^{n-\alpha} \mid \int_{0}^{t}(t-s)^{\alpha-1} \mathbf{E}_{\alpha, \alpha}\left(\lambda(t-s)^{\alpha}\right)[f(s, x(s))-\phi(s)] d s \\
+\sum_{\nu=1}^{n} \sum_{k=1}^{j}\left(t-t_{k}\right)^{\alpha-\nu} \mathbf{E}_{\alpha, \alpha-\nu+1}\left(\lambda\left(t-t_{k}\right)^{\alpha}\right)\left|I_{\nu}\left(t_{k}, x\left(t_{k}\right)\right)-C_{k}\right| \mid \\
\leq\left(t-t_{j}\right)^{n-\alpha} \int_{0}^{t}(t-s)^{\alpha-1} \mathbf{E}_{\alpha, \alpha}(|\lambda|)[r+|| \Phi||]^{\sigma} d s+\sum_{\nu=1}^{n} \sum_{k=1}^{j} \mathbf{E}_{\alpha, \alpha-\nu+1}(|\lambda|)[r+\| \Phi||]^{\sigma} \\
\leq\left[\frac{\mathbf{E}_{\alpha, \alpha}(|\lambda|)}{\alpha}+m \sum_{\nu=1}^{n} \mathbf{E}_{\alpha, \alpha-\nu+1}(|\lambda|)\right][r+\| \Phi||]^{\sigma}
\end{gathered}
$$

Case 1. $\sigma \in[0,1)$. It is easy to see that there exists $r>0$ such that

$$
\left[\frac{\mathbf{E}_{\alpha, \alpha}(|\lambda|)}{\alpha}+m \sum_{\nu=1}^{n} \mathbf{E}_{\alpha, \alpha-\nu+1}(|\lambda|)\right][r+\| \Phi||]^{\sigma} \leq r .
$$


Case 2. $\sigma=1$. It is easy to see that there exists $r>0$ such tha

$$
\left[\frac{\mathbf{E}_{\alpha, \alpha}(|\lambda|)}{\alpha}+m \sum_{\nu=1}^{n} \mathbf{E}_{\alpha, \alpha-\nu+1}(|\lambda|)\right][r+\| \Phi||]^{\sigma} \leq r
$$

by

$$
\frac{\mathbf{E}_{\alpha, \alpha}(|\lambda|)}{\alpha}+m \sum_{\nu=1}^{n} \mathbf{E}_{\alpha, \alpha-\nu+1}(|\lambda|)<1
$$

Case 3. $\sigma>1$. Choose $r=\frac{\|\Phi\|}{\sigma-1}>0$. By

$$
\left[\frac{\mathbf{E}_{\alpha, \alpha}(|\lambda|)}{\alpha}+m \sum_{\nu=1}^{n} \mathbf{E}_{\alpha, \alpha-\nu+1}(|\lambda|)\right]\left(\frac{\sigma}{\sigma-1}\right)^{\sigma}\|\Phi\|^{\sigma-1}<\frac{1}{\sigma-1},
$$

we know that

$$
\left[\frac{\mathbf{E}_{\alpha, \alpha}(|\lambda|)}{\alpha}+m \sum_{\nu=1}^{n} \mathbf{E}_{\alpha, \alpha-\nu+1}(|\lambda|)\right][r+\| \Phi||]^{\sigma} \leq r .
$$

From above discussion, we know $T_{1} \Omega_{r} \subset \Omega_{r}$. Then Schauder's fixed point theorem implies that $T_{1}$ has a fixed point in $\Omega_{r}$. Thus IVP (1.7) has a solution by Theorem 2.5. The proof is completed.

Theorem 3.2. Suppose that there exist non-decreasing functions $\phi_{f}, \phi_{I}:[0, \infty) \rightarrow$ $[0, \infty)$ such that

$$
\begin{aligned}
& \left|f\left(t,\left(t-t_{i}\right)^{\alpha-n} u\right)\right| \leq \phi_{f}(|u|), t \in\left(t_{i}, t_{i+1}\right], \quad i \in \mathbb{N}_{0}^{m}, u \in \mathbb{R}, \\
& \left|I_{j}\left(t_{i},\left(t_{i}-t_{i-1}\right)^{\alpha-n} u\right)\right| \leq \phi_{i}(|u|), \quad i \in \mathbb{N}_{1}^{m}, u \in \mathbb{R} .
\end{aligned}
$$

Then VP (1.8) has at least one solution if there exists $r>0$ such that

$$
\begin{gathered}
{\left[\frac{1}{\Gamma(\alpha+1)}+\frac{1}{|M|} \frac{1}{\Gamma(\alpha-n+2)}\left|\int_{0}^{1}\left(\int_{u}^{1} \frac{(s-u)^{\alpha-1}}{\Gamma(\alpha)} d H(s)-\frac{(1-u)^{\alpha-1}}{\Gamma(\alpha)}\right)\right|\right] \phi_{f}(r)} \\
+\left[\frac{1}{|M|} \frac{1}{\Gamma(\alpha-n+2)}\left|\sum_{\nu=1}^{n} \sum_{k=1}^{m}\left(\int_{t_{k}}^{1} \frac{\left(s-t_{k}\right)^{\alpha-\nu}}{\Gamma(\alpha-\nu+1)} d H(s)-\frac{\left(1-t_{k}\right)^{\alpha-\nu}}{\Gamma(\alpha-\nu+1)}\right)\right|\right. \\
\left.+\sum_{\nu=1}^{n} \frac{m}{\Gamma(\alpha-\nu+1)}\right] \phi_{I}(r) \leq r .
\end{gathered}
$$

Proof. For $r>0$, denote $\Omega_{r}=\left\{x \in P C_{n-\alpha}(0,1]:\|x\| \leq r\right\}$. We will seek $r>0$ such that $T_{2} \Omega_{r} \subseteq \Omega_{r}$. Then Schauder's fixed point theorem implies that $T_{2}$ has a fixed point in $\Omega_{r}$. Thus $\operatorname{VP}(1.6) 8$ has a solution by Theorem 2.5.

For $x \in \Omega_{r}$, we have

$$
\begin{aligned}
|f(t, x(t))|=\mid f(t & \left.\left.\left(t-t_{i}\right)^{\alpha-n}\left(t-t_{i}\right)^{n-\alpha} x(t)\right)\left|\leq \phi_{f}\left(\mid\left(t-t_{i}\right)^{n-\alpha} x(t)\right)\right|\right) \\
& \leq \phi_{f}(\|x\|), t \in\left(t_{i}, t_{i+1}\right], i \in \mathbb{N}_{0}^{m} \\
& \left|I_{j}\left(t_{i}, x\left(t_{i}\right)\right)\right| \leq \phi_{i}(\|x\|), i \in \mathbb{N}_{1}^{m} .
\end{aligned}
$$

For $t \in\left(t_{j}, t_{j+1}\right]$, we have

$$
\left(t-t_{j}\right)^{n-\alpha}\left|\left(T_{2} x\right)(t)\right|=\left(t-t_{j}\right)^{n-\alpha} \mid \int_{0}^{t} \frac{(t-s)^{\alpha-1}}{\Gamma(\alpha)} f(s, x(s)) d s
$$




$$
\begin{aligned}
& +\frac{1}{M}\left[\int_{0}^{1}\left(\int_{u}^{1} \frac{(s-u)^{\alpha-1}}{\Gamma(\alpha)} d H(s)-\frac{(1-u)^{\alpha-1}}{\Gamma(\alpha)}\right) f(u, x(u)) d u\right. \\
& \left.+\sum_{\nu=1}^{n} \sum_{k=1}^{m}\left(\int_{t_{k}}^{1} \frac{\left(s-t_{k}\right)^{\alpha-\nu}}{\Gamma(\alpha-\nu+1)} d H(s)-\frac{\left(1-t_{k}\right)^{\alpha-\nu}}{\Gamma(\alpha-\nu+1)}\right) I_{\nu}\left(t_{k}, x\left(t_{k}\right)\right)\right] \frac{t^{\alpha-n+1}}{\Gamma(\alpha-n+2)} \\
& +\sum_{\nu=1}^{n} \sum_{k=1}^{j} \frac{\left(t-t_{k}\right)^{\alpha-\nu}}{\Gamma(\alpha-\nu+1)} I_{\nu}\left(t_{k}, x\left(t_{k}\right)\right) \\
& \leq\left(t-t_{j}\right)^{n-\alpha} \int_{0}^{t} \frac{(t-s)^{\alpha-1}}{\Gamma(\alpha)} \phi_{f}(\|x\|) d s \\
& +\frac{1}{|M|} \frac{1}{\Gamma(\alpha-n+2)} \int_{0}^{1}\left(\int_{u}^{1} \frac{(s-u)^{\alpha-1}}{\Gamma(\alpha)} d H(s)-\frac{(1-u)^{\alpha-1}}{\Gamma(\alpha)}\right) \phi_{f}(\|x\|) d u \\
& +\frac{1}{|M|} \frac{1}{\Gamma(\alpha-n+2)} \sum_{\nu=1}^{n} \sum_{k=1}^{m}\left(\int_{t_{k}}^{1} \frac{\left(s-t_{k}\right)^{\alpha-\nu}}{\Gamma(\alpha-\nu+1)} d H(s)-\frac{\left(1-t_{k}\right)^{\alpha-\nu}}{\Gamma(\alpha-\nu+1)}\right) \phi_{I}(\|x\|) \\
& +\left(t-t_{j}\right)^{n-\alpha} \sum_{\nu=1}^{n} \sum_{k=1}^{j} \frac{\left(t-t_{k}\right)^{\alpha-\nu}}{\Gamma(\alpha-\nu+1)} \phi_{I}(\|x\|) \\
& \leq \frac{1}{\Gamma(\alpha+1)} \phi_{f}(\|x\|) \\
& +\frac{1}{|M|} \frac{1}{\Gamma(\alpha-n+2)}\left|\int_{0}^{1}\left(\int_{u}^{1} \frac{(s-u)^{\alpha-1}}{\Gamma(\alpha)} d H(s)-\frac{(1-u)^{\alpha-1}}{\Gamma(\alpha)}\right)\right| \phi_{f}(\|x\|) \\
& +\frac{1}{|M|} \frac{1}{\Gamma(\alpha-n+2)}\left|\sum_{\nu=1}^{n} \sum_{k=1}^{m}\left(\int_{t_{k}}^{1} \frac{\left(s-t_{k}\right)^{\alpha-\nu}}{\Gamma(\alpha-\nu+1)} d H(s)-\frac{\left(1-t_{k}\right)^{\alpha-\nu}}{\Gamma(\alpha-\nu+1)}\right)\right| \phi_{I}(\|x\|) \\
& +\sum_{\nu=1}^{n} \frac{m}{\Gamma(\alpha-\nu+1)} \phi_{I}(\|x\|) \\
& \leq\left[\frac{1}{\Gamma(\alpha+1)}+\frac{1}{|M|} \frac{1}{\Gamma(\alpha-n+2)}\left|\int_{0}^{1}\left(\int_{u}^{1} \frac{(s-u)^{\alpha-1}}{\Gamma(\alpha)} d H(s)-\frac{(1-u)^{\alpha-1}}{\Gamma(\alpha)}\right)\right|\right] \phi_{f}(r) \\
& +\left[\frac{1}{|M|} \frac{1}{\Gamma(\alpha-n+2)}\left|\sum_{\nu=1}^{n} \sum_{k=1}^{m}\left(\int_{t_{k}}^{1} \frac{\left(s-t_{k}\right)^{\alpha-\nu}}{\Gamma(\alpha-\nu+1)} d H(s)-\frac{\left(1-t_{k}\right)^{\alpha-\nu}}{\Gamma(\alpha-\nu+1)}\right)\right|\right. \\
& \left.+\sum_{\nu=1}^{n} \frac{m}{\Gamma(\alpha-\nu+1)}\right] \phi_{I}(r)
\end{aligned}
$$

By the assumption of theorem, we have $\left(t-t_{j}\right)^{n-\alpha}\left|\left(T_{2} x\right)(t)\right| \leq r$ for all $t \in\left(t_{i}, t_{i+1}\right]$, $i \in \mathbb{N}_{0}^{m}$. Then $\left\|T_{2} x\right\| \leq r$. Hence $T_{2} \Omega_{r} \subset \Omega_{r}$. The proof is completed. 


\section{Comments on published paprs}

We have the following result:

Theorem 4.1. Consider the homogenous form of $B V P(1.7)$ :

$$
\left\{\begin{array}{l}
D_{0^{+}}^{\alpha} x(t)-\lambda x(t)=0, t \in\left(t_{i}, t_{i+1}\right], i \in \mathbb{N}_{0}^{m}, \\
\Delta I_{0^{+}}^{\beta} x\left(t_{i}\right)=: I_{0^{+}}^{\beta} x\left(t_{i}^{+}\right)-I_{0^{+}}^{\beta} x\left(t_{i}\right)=0, i \in \mathbb{N}_{1}^{m}, \\
\Delta D_{0^{+}}^{\alpha-j} x\left(t_{i}\right)=: D_{0^{+}}^{\alpha-j} x\left(t_{i}^{+}\right)-D_{0^{+}}^{\alpha-j} x\left(t_{i}\right)=0, i \in \mathbb{N}_{1}^{m}, j \in \mathbb{N}_{1}^{n-1}, \\
I_{0^{+}}^{n-\alpha} x(0)=D_{0^{+}}^{\alpha-j} x(0)=0, j \in \mathbb{N}_{1}^{n-1} .
\end{array}\right.
$$

Then IVP (4.1) has infinitely many solutions if $\alpha+\beta>n$ and IVP (4.1) has a unique solution $x(t)=0$ if $\alpha+\beta=n$.

Proof. By Theorem 2.1 and $D_{0^{+}}^{\alpha} x(t)-\lambda x(t)=0, t \in\left(t_{i}, t_{i+1}\right], i \in \mathbb{N}_{0}^{m}$, we get that there exist constants $c_{\nu k} \in \mathbb{R}$ such that

$$
x(t)=\sum_{k=0}^{i} \sum_{\nu=1}^{n} c_{\nu k}\left(t-t_{k}\right)^{\alpha-\nu} \mathbf{E}_{\alpha, \alpha-\nu+1}\left(\lambda\left(t-t_{k}\right)^{\alpha}\right), t \in\left(t_{i}, t_{i+1}\right], i \in \mathbb{N}_{0}^{m} .
$$

By Theorem 2.2, we get

$$
\begin{gathered}
I_{0^{+}}^{n-\alpha} x(t) \sum_{k=0}^{i} \sum_{\nu=1}^{n} c_{\nu k}\left(t-t_{k}\right)^{n-\nu} \mathbf{E}_{\alpha, n-\nu+1}\left(\lambda\left(t-t_{k}\right)^{\alpha}\right), t \in\left(t_{i}, t_{i+1}\right], i \in \mathbb{N}_{0}^{m}, \\
I_{0^{+}}^{\beta} x(t)=\sum_{k=0}^{i} \sum_{\nu=1}^{n} c_{\nu k}\left(t-t_{k}\right)^{\alpha+\beta-\nu} \mathbf{E}_{\alpha, \alpha+\beta-\nu+1}\left(\lambda\left(t-t_{k}\right)^{\alpha}\right), t \in\left(t_{i}, t_{i+1}\right], i \in \mathbb{N}_{0}^{m}, \\
D_{0^{+}}^{\alpha-j} x(t)=\sum_{k=0}^{i} \sum_{\nu=1}^{j} c_{\nu k}\left(t-t_{k}\right)^{j-\nu} \mathbf{E}_{\alpha, j-\nu+1}\left(\lambda\left(t-t_{k}\right)^{\alpha}\right) \\
+\lambda \sum_{k=0}^{i} \sum_{\nu=j+1}^{n} c_{\nu k}\left(t-t_{k}\right)^{\alpha+j-\nu} \mathbf{E}_{\alpha, \alpha+j-\nu+1}\left(\lambda\left(t-t_{k}\right)^{\alpha}\right), t \in\left(t_{i}, t_{i+1}\right], i \in \mathbb{N}_{0}^{m}, j \in \mathbb{N}_{1}^{n-1} .
\end{gathered}
$$

(i) By $I_{0^{+}}^{n-\alpha} x(0)=0$ and the expression of $I_{0^{+}}^{n-\alpha} x$, we get $c_{n 0}=0$.

(ii) By $D_{0^{+}}^{\alpha-j} x(0)=0, j \in \mathbb{N}_{1}^{n-1}$ and and the expression of $D_{0^{+}}^{\alpha-j} x$, we get $c_{j 0}=0$ for all $j \in \mathbb{N}_{1}^{n-1}$.

(iii) By $\Delta D_{0^{+}}^{\alpha-j} x\left(t_{i}\right)=0, i \in \mathbb{N}_{1}^{m}, j \in \mathbb{N}_{1}^{n-1}$ and the expression of $D_{0^{+}}^{\alpha-j} x$, we get $c_{j i}=0$ for all $i \in \mathbb{N}_{1}^{m}, j \in \mathbb{N}_{1}^{n-1}$.

Then

$$
x(t)=\sum_{k=1}^{i} c_{n k}\left(t-t_{k}\right)^{\alpha-n} \mathbf{E}_{\alpha, \alpha-n+1}\left(\lambda\left(t-t_{k}\right)^{\alpha}\right), t \in\left(t_{i}, t_{i+1}\right], i \in \mathbb{N}_{0}^{m}
$$

and

$$
I_{0^{+}}^{\beta} x(t)=\sum_{k=1}^{i} c_{n k}\left(t-t_{k}\right)^{\alpha+\beta-n} \mathbf{E}_{\alpha, \alpha+\beta-n+1}\left(\lambda\left(t-t_{k}\right)^{\alpha}\right), t \in\left(t_{i}, t_{i+1}\right], i \in \mathbb{N}_{0}^{m} .
$$


Case 1. $\alpha+\beta=n$.

By $\Delta I_{0^{+}}^{\beta} x\left(t_{i}\right)=: I_{0^{+}}^{\beta} x\left(t_{i}^{+}\right)-I_{0^{+}}^{\beta} x\left(t_{i}\right)=0, i \in \mathbb{N}_{1}^{m}$, we get $c_{n i}=0$ for all $i \in \mathbb{N}_{1}^{m}$. It follows that $x(t)=0$ is a unique solution.

Case 2. $\alpha+\beta>n$.

By $\Delta I_{0^{+}}^{\beta} x\left(t_{i}\right)=: I_{0^{+}}^{\beta} x\left(t_{i}^{+}\right)-I_{0^{+}}^{\beta} x\left(t_{i}\right)=0, i \in \mathbb{N}_{1}^{m}$, we get

$$
-\sum_{k=1}^{i-1} c_{n k}\left(t_{i}-t_{k}\right)^{\alpha+\beta-n} \mathbf{E}_{\alpha, \alpha+\beta-n+1}\left(\lambda\left(t_{i}-t_{k}\right)^{\alpha}\right)=0, i \in \mathbb{N}_{1}^{m}
$$

Hence $c_{n i}=0$ for all $i \in \mathbb{N}_{1}^{m-1}$.

Then

$$
x(t)=\left\{\begin{array}{l}
0, t \in\left(t_{i}, t_{i+1}\right], i \in \mathbb{N}_{0}^{m-1}, \\
c_{n m}\left(t-t_{m}\right)^{\alpha-n} \mathbf{E}_{\alpha, \alpha-n+1}\left(\lambda\left(t-t_{m}\right)^{\alpha}\right), t \in\left(t_{m}, t_{m+1}\right] .
\end{array}\right.
$$

Here $c_{n m} \in \mathbb{R}$ is a constants. Hence it has infinitely many solutions defined.

In [8], Kosmatov studied the solvability of IVP (1.1).

Define the operator $T_{\alpha}: P C_{\alpha}(0,] \rightarrow P C_{\alpha}(0,1]$ by

$$
\begin{aligned}
\left(T_{\alpha} x\right)(t) & =\frac{x_{0}}{\Gamma(\alpha)} t^{\alpha-1}+\frac{\Gamma(\alpha-\beta)}{\Gamma(\alpha)}\left(\sum_{0<t_{k}<t} t_{k}^{1+\beta-\alpha} J_{k}\left(x\left(t_{k}\right)\right)\right) t^{\alpha-1} \\
& +\int_{0}^{t} \frac{(t-s)^{\alpha-1}}{\Gamma(\alpha)} f(s, x(s)) d s, t \in(0,1] .
\end{aligned}
$$

Result 4.1. (see page 1296 in [8]). $x$ is a solution of (1.1) if and only if $x$ is a fixed point of $T_{\alpha}$ in $P C_{\alpha}(0,1]$.

Remark 4.1. By Lemma $2.1(n=1, \lambda=0), x$ is a solution of $D_{0^{+}}^{\alpha} x(t)=f(t, x(t))$, $t \in[0,1] \backslash\left\{t_{1}, t_{2}, \cdots, t_{m}\right\}$ if and only if there exist constants $c_{i} \in \mathbb{R}$ such that

$$
x(t)=\sum_{j=0}^{i} c_{j}\left(t-t_{j}\right)^{\alpha-1}+\int_{0}^{t} \frac{(t-s)^{\alpha-1}}{\Gamma(\alpha)} f(s, x(s)) d s, t \in\left(t_{i}, t_{i+1}\right], i \in \mathbb{N}_{0}^{m} .
$$

We can get by direct computation that

$$
\begin{gathered}
D_{0^{+}}^{\beta} x(t)=\sum_{j=0}^{i} \frac{\Gamma(\alpha)}{\Gamma(\alpha-\beta)} c_{j}\left(t-t_{j}\right)^{\alpha-\beta-1}+\int_{0}^{t} \frac{(t-s)^{\alpha-\beta-1}}{\Gamma(\alpha-\beta)} f(s, x(s)) d s, \\
t \in\left(t_{i}, t_{i+1}\right], i \in \mathbb{N}_{0}^{m} .
\end{gathered}
$$

By $\beta \in(0, \alpha)$, we know $\alpha-\beta-1<0$. We find that $D_{0^{+}}^{\beta} x(t)$ is singular at $t=t_{i}$. Hence the impulse functions are unsuitable.

From above discussion, we know that Result 4.1 [8] is unsuitable.

Result 4.2. (Lemma 2.7 in [14]). Suppose that $q, \alpha \in(0,1)$. Then $x$ is a solution of (1.3) if and only if $x$ is a fixed point of the operator $T_{q}: P C_{q}(0,1] \mapsto P C_{q}(0,1]$, where $T_{q}$ is defined by

$$
\left(T_{q} x\right)(t)=\frac{\Gamma(q) t^{q-1} \mathbf{E}_{q, q}\left(-\lambda_{0} t^{q}\right)}{1+\Gamma(q) \mathbf{E}_{q, q}\left(-\lambda_{0}\right)}
$$




$$
\begin{gathered}
\times\left[\sum_{i=1}^{m} \frac{J_{i}\left(x\left(t_{i}\right)\right)}{\Gamma(q) t_{i}^{\alpha+q-1} \mathbf{E}_{q, q+\alpha}\left(-\lambda_{0} t_{i}^{q}\right)}-\int_{0}^{1}(1-s)^{q-1} \mathbf{E}_{q, q}\left(-\lambda_{0}(1-s)^{q}\right) f(s, x(s)) d s\right] \\
-t^{q-1} \mathbf{E}_{q, q}\left(-\lambda_{0} t^{q}\right) \sum_{t \leq t_{i}<1} \frac{J_{i}\left(x\left(t_{i}\right)\right)}{t_{i}^{\alpha+q-1} \mathbf{E}_{q, q+\alpha}\left(-\lambda_{0} t_{i}^{q}\right)} \\
+\int_{0}^{t}(t-s)^{q-1} \mathbf{E}_{q, q}\left(-\lambda_{0}(t-s)^{q}\right) f(s, x(s)) d s .
\end{gathered}
$$

Remark 4.2. Result 4.2 is incorrect.

Proof. By Lemma $2.1((n=1))$, if $x$ is a solution of BVP (4.3), then there exists constants $c_{i}\left(i \in \mathbf{N}_{0}^{m}\right)$ such that

$$
\begin{aligned}
x(t) & =\sum_{v=0}^{j} c_{v}\left(t-t_{v}\right)^{q-1} \mathbf{E}_{q, q}\left(-\lambda_{0}\left(t-t_{v}\right)^{q}\right) \\
& +\int_{0}^{t}(t-s)^{q-1} \mathbf{E}_{q, q}\left(-\lambda_{0}(t-s)^{q}\right) f(s, x(s)) d s, t \in\left(t_{j}, t_{j+1}\right], j \in \mathbf{N}_{0}^{m} .
\end{aligned}
$$

By Definition 2.1, we get for $t \in\left(t_{j}, t_{j+1}\right]$ that

$$
\begin{aligned}
I_{0^{+}}^{\alpha} x(t)= & \int_{0}^{t} \frac{(t-s)^{\alpha-1}}{\Gamma(\alpha)} x(s) d s=\frac{\sum_{\tau=0}^{j-1} \int_{t_{\tau}}^{t_{\tau+1}}(t-s)^{\alpha-1} x(s) d s+\int_{t_{j}}^{t}(t-s)^{\alpha-1} x(s) d s}{\Gamma(\alpha)} \\
= & \left(\sum _ { \tau = 0 } ^ { j - 1 } \int _ { t _ { \tau } } ^ { t _ { \tau + 1 } } ( t - s ) ^ { \alpha - 1 } \left[\sum_{v=0}^{\tau} c_{v}\left(s-t_{v}\right)^{q-1} \mathbf{E}_{q, q}\left(-\lambda_{0}\left(s-t_{v}\right)^{q}\right)\right.\right. \\
& \left.\left.+\int_{0}^{s}(s-u)^{q-1} \mathbf{E}_{q, q}\left(-\lambda_{0}(s-u)^{q}\right) f(u, x(u)) d u\right] d s\right) / \Gamma(\alpha) \\
& +\left(\int _ { t _ { j } } ^ { t } ( t - s ) ^ { \alpha - 1 } \left[\sum_{v=0}^{j} c_{v}\left(s-t_{v}\right)^{q-1} \mathbf{E}_{q, q}\left(-\lambda_{0}\left(s-t_{v}\right)^{q}\right)\right.\right. \\
& \left.\left.+\int_{0}^{s}(s-u)^{q-1} \mathbf{E}_{q, q}\left(-\lambda_{0}(s-u)^{q}\right) f(u, x(u)) d u\right] d s\right) / \Gamma(\alpha) \\
& \sum_{\tau=0}^{j-1} \sum_{v=0}^{\tau} c_{v} \int_{t_{\tau}}^{t_{\tau+1}}(t-s)^{\alpha-1}\left(s-t_{v}\right)^{q-1} \mathbf{E}_{q, q}\left(-\lambda_{0}\left(s-t_{v}\right)^{q}\right) d s \\
= & \frac{\Gamma(\alpha)}{}
\end{aligned}
$$

change the order of sum and integral

$$
\begin{gathered}
+\left(\sum_{v=0}^{j} c_{v} \int_{t_{j}}^{t}(t-s)^{\alpha-1}\left(s-t_{v}\right)^{q-1} \mathbf{E}_{q, q}\left(-\lambda_{0}\left(s-t_{v}\right)^{q}\right) d s\right. \\
\left.+\int_{0}^{t}(t-s)^{\alpha-1} \int_{0}^{s}(s-u)^{q-1} \mathbf{E}_{q, q}\left(-\lambda_{0}(s-u)^{q}\right) f(u, x(u)) d u d s\right) / \Gamma(\alpha) \\
=\frac{\sum_{v=0}^{j-1} \sum_{\tau=v}^{j-1} c_{v} \int_{t_{\tau}}^{t_{\tau+1}}(t-s)^{\alpha-1}\left(s-t_{v}\right)^{q-1} \mathbf{E}_{q, q}\left(-\lambda_{0}\left(s-t_{v}\right)^{q}\right) d s}{\Gamma(\alpha)}
\end{gathered}
$$


change the order of sum

$$
\begin{gathered}
+\left(\sum_{v=0}^{j} c_{v} \int_{t_{j}}^{t}(t-s)^{\alpha-1}\left(s-t_{v}\right)^{q-1} \mathbf{E}_{q, q}\left(-\lambda_{0}\left(s-t_{v}\right)^{q}\right) d s\right. \\
\left.+\int_{0}^{t} \int_{u}^{t}(t-s)^{\alpha-1}(s-u)^{q-1} \mathbf{E}_{q, q}\left(-\lambda_{0}(s-u)^{q}\right) d s f(u, x(u)) d u\right) / \Gamma(\alpha)
\end{gathered}
$$

change the order of integral

$$
\begin{gathered}
=\left(\sum_{v=0}^{j} c_{v} \int_{t_{v}}^{t}(t-s)^{\alpha-1}\left(s-t_{v}\right)^{q-1} \mathbf{E}_{q, q}\left(-\lambda_{0}\left(s-t_{v}\right)^{q}\right) d s\right. \\
\left.+\int_{0}^{t} \int_{u}^{t}(t-s)^{\alpha-1}(s-u)^{q-1} \mathbf{E}_{q, q}\left(-\lambda_{0}(s-u)^{q}\right) d s f(u, x(u)) d u\right) / \Gamma(\alpha) \\
=\left(\sum_{v=0}^{j} c_{v} \sum_{\chi=0}^{\infty} \frac{\left(-\lambda_{0}\right)^{\chi}}{\Gamma(\chi q+q)} \int_{t_{v}}^{t}(t-s)^{\alpha-1}\left(s-t_{v}\right)^{\chi q+q-1} d s\right. \\
\left.+\int_{0}^{t} \sum_{\chi=0}^{\infty} \frac{\left(-\lambda_{0}\right)^{\chi}}{\Gamma(\chi q+q)} \int_{u}^{t}(t-s)^{\alpha-1}(s-u)^{\chi q+q-1} d s f(u, x(u)) d u\right) / \Gamma(\alpha)
\end{gathered}
$$

by

$$
\begin{gathered}
\frac{s-t_{v}}{t-t_{v}}=w, \quad \frac{s-u}{t-u}=w \\
=\frac{\sum_{v=0}^{j} c_{v} \sum_{\chi=0}^{\infty} \frac{\left(-\lambda_{0}\right)^{\chi}\left(t-t_{v}\right)^{\chi q+\alpha+q-1}}{\Gamma(\chi q+q)} \int_{0}^{1}(1-w)^{\alpha-1} w^{\chi q+q-1} d w}{\Gamma(\alpha)} \\
+\frac{\int_{0}^{t} \sum_{\chi=0}^{\infty} \frac{\left(-\lambda_{0}\right)^{\chi}(t-u)^{\chi q+\alpha+q-1}}{\Gamma(\chi q+q)} \int_{0}^{1}(1-w)^{\alpha-1} w^{\chi q+q-1} d w f(u, x(u)) d u}{\Gamma(\alpha)} \\
=\sum_{v=0}^{j} c_{v=0}^{\infty} \frac{\left(-\lambda_{0}\right)^{\chi}\left(t-t_{v}\right)^{\chi q+\alpha+q-1}}{\Gamma(\chi q+\alpha+q)} \\
+\int_{0}^{t} \sum_{\chi=0}^{\infty} \frac{\left(-\lambda_{0}\right)^{\chi}(t-u)^{\chi q+\alpha+q-1}}{\Gamma(\chi q+\alpha+q)} f(u, x(u)) d u \\
=\sum_{v=0}^{t} c_{0}^{j}(t-u)^{\alpha+q-1} \mathbf{E}_{q, \alpha+q}\left(-\lambda_{0}(t-u)^{q}\right) f(u, x(u)) d u .
\end{gathered}
$$


It follows that

$$
\begin{aligned}
I_{0^{+}}^{\alpha} x(t)= & \sum_{v=0}^{j} c_{v}\left(t-t_{v}\right)^{\alpha+q-1} \mathbf{E}_{q, \alpha+q}\left(-\lambda_{0}\left(t-t_{v}\right)^{q}\right) \\
+ & \int_{0}^{t}(t-u)^{\alpha+q-1} \mathbf{E}_{q, \alpha+q}\left(-\lambda_{0}(t-u)^{q}\right) f(u, x(u)) d u, \\
& t \in\left(t_{j}, t_{j+1}\right], j \in \mathbf{N}_{0}^{m} .
\end{aligned}
$$

Case 1. $\alpha+q<1$. From $I_{0^{+}}^{\alpha} x\left(t_{i}^{+}\right)-I_{0^{+}}^{\alpha}\left(t_{i}^{-}\right)=J_{i}\left(x\left(t_{i}\right)\right), i=1,2, \cdots, m$, and (14.11), we get $J_{i}\left(x\left(t_{i}\right)\right)=\infty\left(i \in \mathbf{N}_{1}^{m}\right)$. This is a contradiction. So BVP (4.2) is unsuitable proposed.

Case 2. $\alpha+q=1$. From $I_{0^{+}}^{\alpha} x\left(t_{i}^{+}\right)-I_{0^{+}}^{\alpha}\left(t_{i}^{-}\right)=J_{i}\left(x\left(t_{i}\right)\right), i=1,2, \cdots, m$, and (14.11), we get $J_{i}\left(x\left(t_{i}\right)\right)=c_{i}\left(i \in \mathbb{N}_{1}^{m}\right)$. From $\left.t^{1-q} x(t)\right|_{t=0}+\left.t^{1-q} x(t)\right|_{t=1}=0$ and (14.10), we get

$c_{0}+\sum_{v=0}^{m} c_{v}\left(1-t_{v}\right)^{q-1} \mathbf{E}_{q, q}\left(-\lambda_{0}\left(1-t_{v}\right)^{q}\right)+\int_{0}^{1}(1-s)^{q-1} \mathbf{E}_{q, q}\left(-\lambda_{0}(1-s)^{q}\right) f(s, x(s)) d s=0$.

Then

$$
\begin{gathered}
c_{0}=-\left(\sum_{v=1}^{m} J_{v}\left(x\left(t_{v}\right)\right)\left(1-t_{v}\right)^{q-1} \mathbf{E}_{q, q}\left(-\lambda_{0}\left(1-t_{v}\right)^{q}\right)\right. \\
\left.+\int_{0}^{1}(1-s)^{q-1} \mathbf{E}_{q, q}\left(-\lambda_{0}(1-s)^{q}\right) f(s, x(s)) d s\right) /\left(1+\mathbf{E}_{q, q}\left(-\lambda_{0}\right)\right) .
\end{gathered}
$$

Hence $x$ is a solution of BVP (4.2) if and only if

$$
\begin{gathered}
x(t)=-\left(\sum_{v=1}^{m} J_{v}\left(x\left(t_{v}\right)\right)\left(1-t_{v}\right)^{q-1} \mathbf{E}_{q, q}\left(-\lambda_{0}\left(1-t_{v}\right)^{q}\right)\right. \\
\left.+\int_{0}^{1}(1-s)^{q-1} \mathbf{E}_{q, q}\left(-\lambda_{0}(1-s)^{q}\right) f(s, x(s)) d s\right) /\left(1+\mathbf{E}_{q, q}\left(-\lambda_{0}\right)\right) t^{q-1} \mathbf{E}_{q, q}\left(-\lambda_{0} t^{q}\right) \\
+\sum_{v=1}^{j} I_{v}\left(x\left(t_{v}\right)\right)\left(t-t_{v}\right)^{q-1} \mathbf{E}_{q, q}\left(-\lambda_{0}\left(t-t_{v}\right)^{q}\right) \\
+\int_{0}^{t}(t-s)^{q-1} \mathbf{E}_{q, q}\left(-\lambda_{0}(t-s)^{q}\right) f(s, x(s)) d s, t \in\left(t_{j}, t_{j+1}\right], j \in \mathbf{N}_{0}^{m} .
\end{gathered}
$$

Case 3. $\alpha+q>1$. From $I_{0^{+}}^{\alpha} x\left(t_{i}^{+}\right)-I_{0^{+}}^{\alpha}\left(t_{i}^{-}\right)=J_{i}\left(x\left(t_{i}\right)\right), i=1,2, \cdots, m$, and (14.11), we get $J_{i}\left(x\left(t_{i}\right)\right)=0\left(i \in \mathbb{N}_{1}^{m}\right)$. From $\left.t^{1-q} x(t)\right|_{t=0}+\left.t^{1-q} x(t)\right|_{t=1}=0$ and (14.10), we get similarly as in Case 2 that

$c_{0}=-\frac{\sum_{v=1}^{m} c_{v}\left(1-t_{v}\right)^{q-1} \mathbf{E}_{q, q}\left(-\lambda_{0}\left(1-t_{v}\right)^{q}\right)+\int_{0}^{1}(1-s)^{q-1} \mathbf{E}_{q, q}\left(-\lambda_{0}(1-s)^{q}\right) f(s, x(s)) d s}{1+\mathbf{E}_{q, q}\left(-\lambda_{0}\right)}$. 
Hence $x$ is a solution of BVP (4.2) if and only if

$$
\begin{gathered}
x(t)=-\frac{\sum_{v=1}^{m} c_{v}\left(1-t_{v}\right)^{q-1} \mathbf{E}_{q, q}\left(-\lambda_{0}\left(1-t_{v}\right)^{q}\right)+\int_{0}^{1}(1-s)^{q-1} \mathbf{E}_{q, q}\left(-\lambda_{0}(1-s)^{q}\right) f(s, x(s)) d s}{1+\mathbf{E}_{q, q}\left(-\lambda_{0}\right)} \\
\times t^{q-1} \mathbf{E}_{q, q}\left(-\lambda_{0} t^{q}\right)+\sum_{v=1}^{j} c_{v}\left(t-t_{v}\right)^{q-1} \mathbf{E}_{q, q}\left(-\lambda_{0}\left(t-t_{v}\right)^{q}\right) \\
+\int_{0}^{t}(t-s)^{q-1} \mathbf{E}_{q, q}\left(-\lambda_{0}(t-s)^{q}\right) f(s, x(s)) d s, t \in\left(t_{j}, t_{j+1}\right], j \in \mathbf{N}_{0}^{m} .
\end{gathered}
$$

Then $x$ is a solution of BVP (4.2) if and only if

$$
\begin{gathered}
x(t)=-\frac{\sum_{v=1}^{m} c_{v}\left(t-t_{v}\right)^{q-1} \mathbf{E}_{q, q}\left(-\lambda_{0}\left(1-t_{v}\right)^{q}\right)+\int_{0}^{1}(1-s)^{q-1} \mathbf{E}_{q, q}\left(-\lambda_{0}(1-s)^{q}\right) f(s, x(s)) d s}{1+\mathbf{E}_{q, q}\left(-\lambda_{0}\right)} \\
\times t^{q-1} \mathbf{E}_{q, q}\left(-\lambda_{0} t^{q}\right)+\sum_{v=1}^{j} c_{v}\left(t-t_{v}\right)^{q-1} \mathbf{E}_{q, q}\left(-\lambda_{0}\left(t-t_{v}\right)^{q}\right) \\
+\int_{0}^{t}(t-s)^{q-1} \mathbf{E}_{q, q}\left(-\lambda_{0}(t-s)^{q}\right) f(s, x(s)) d s, t \in\left(t_{j}, t_{j+1}\right], j \in \mathbf{N}_{0}^{m}
\end{gathered}
$$

and $J_{i}\left(x\left(t_{i}\right)\right)=0\left(i \in \mathbf{N}_{1}^{m}\right)$.

Hence from Case 1-Case 3 Result 4.2 is incorrect.

In [17], Zhao studied the existence of solutions of $\operatorname{BVP}(1.7)$ for the higher-order nonlinear Riemann-Liouville fractional differential equation with Riemann-Stieltjes integral boundary value conditions and impulses Lemma 2.4 [17] claimed:

Result 4.3. If $H$ is a function of bounded variation

$$
\delta=\int_{0}^{1} s^{\alpha-1} d H(s) \alpha-1
$$

and $h \in C([0,1])$, then the unique solution of

$$
\begin{gathered}
-D_{0^{+}}^{\alpha} x(t)=h(t), t \backslash\left\{t_{i}\right\}_{i=1}^{m}, \\
\Delta x\left(t_{i}\right)=I_{i}\left(x\left(t_{i}\right)\right), i=1,2, \cdots, m, \\
x(0)=x^{\prime}(0)=\cdots=x^{(n-2)} 0=0, x^{\prime}(1)=\int_{0}^{1} x(s) d H(s),
\end{gathered}
$$

is

$$
x(t)=\int_{0}^{1} G(t, s) h(s) d s+t^{\alpha-1} \sum_{t \leq t_{k}<1} t_{k}^{1-\alpha} I_{k}\left(x\left(t_{k}\right)\right), t \in[0,1],
$$

where $G(t, s)=G_{1}(t, s)+G_{2}(t, s)$ and

$$
G_{1}(t, s)=\left\{\begin{array}{l}
\frac{t^{\alpha-1}(1-s)^{\alpha-2}-(t-s)^{\alpha-1}}{\Gamma(\alpha)}, 0 \leq s \leq t \leq 1, \\
\frac{t^{\alpha-1}(1-s)^{\alpha-2}}{\Gamma(\alpha)}, \quad 0 \leq t \leq s \leq 1
\end{array}\right.
$$




$$
G_{2}(t, s)=\frac{t^{\alpha-1}}{\alpha-1-\delta} \int_{0}^{1} G_{1}(\tau, s) d H(\tau) .
$$

Remark 4.3. Result 4.3 is wrong.

In fact, we re-write (4.7) by

$$
x(t)=-\int_{0}^{t} \frac{(t-s)^{\alpha-1}}{\Gamma(\alpha)} h(s) d s+M_{k} t^{\alpha-1}, t \in\left(t_{k-1}, t_{k}\right], k \in \mathbb{N}_{1}^{m+1},
$$

where

$$
\begin{aligned}
M_{k} & =\sum_{j=k}^{m} t_{j}^{1-\alpha} I_{j}\left(x\left(t_{j}\right)+\int_{0}^{1} \frac{(1-s)^{\alpha-2}}{\Gamma(\alpha)} h(s) d s\right. \\
& +\frac{1}{\alpha-1-\delta} \int_{0}^{1} \int_{0}^{1} G_{1}(\tau, s) d H(\tau) h(s) d s .
\end{aligned}
$$

One finds from Definition 2.2 for $t \in\left(t_{i}, t_{i+1}\right]$ that

$$
\begin{gathered}
D_{0^{+}}^{\alpha} x(t)=\frac{\left[\int_{0}^{t}(t-s)^{n-\alpha-1} x(s) d s\right]^{(n)}}{\Gamma(n-\alpha)} \\
=\frac{\left[\sum_{\mu=0}^{i-1} \int_{t_{\mu}}^{t_{\mu+1}}(t-s)^{n-\alpha-1} x(s) d s+\int_{t_{i}}^{t}(t-s)^{n-\alpha-1} x(s) d s\right]^{(n)}}{\Gamma(n-\alpha)} \\
=\frac{\left[\sum_{\mu=0}^{i-1} \int_{t_{\mu}}^{t_{\mu+1}}(t-s)^{n-\alpha-1}\left(-\int_{0}^{s} \frac{(s-u)^{\alpha-1}}{\Gamma(\alpha)} h(u) d u+M_{\mu+1} s^{\alpha-1}\right) d s\right]^{(n)}}{\Gamma(n-\alpha)} \\
=\frac{\left[\int_{t_{i}}^{t}(t-s)^{n-\alpha-1}\left(-\int_{0}^{s} \frac{(s-u)^{\alpha-1}}{\Gamma(\alpha)} h(u) d u+M_{i+1} s^{\alpha-1}\right) d s\right]^{(n)}}{\Gamma(n-\alpha)} \\
+\frac{\left[\sum_{\mu=0}^{i-1} M_{\mu+1} \int_{t_{\mu}}^{t_{\mu+1}}(t-s)^{n-\alpha-1} s^{\alpha-1} d s\right]^{(n)}}{\Gamma(n-\alpha)} \\
+\frac{\left[-\int_{0}^{t}(t-s)^{n-\alpha-1} \int_{0}^{s} \frac{(s-u)^{\alpha-1}}{\Gamma(\alpha)} h(t-s)^{n-\alpha-1} \frac{(s-u)^{\alpha-1}}{\Gamma(\alpha)} d s h(u) d u\right]^{(n)}}{\Gamma(n-\alpha)} \\
\left.\left.\sum_{\mu=0}^{i-1} M_{\mu+1} \int_{t_{\mu}}^{t_{\mu+1}}(t-s)^{n-\alpha-1} s^{\alpha-1} d s\right]_{t_{i}}^{t}(t-s)^{n-\alpha-1} s^{\alpha-1} d s\right]^{(n)} \\
\Gamma(n-\alpha)
\end{gathered}
$$




$$
\begin{gathered}
=\frac{\left[\sum_{\mu=0}^{i-1} M_{\mu+1} t^{n-1} \int_{\frac{t_{\mu}}{t}}^{\frac{t_{\mu+1}}{t}}(1-w)^{n-\alpha-1} w^{\alpha-1} d w\right]^{(n)}}{\Gamma(n-\alpha)} \\
+\frac{\left[M_{i+1} \int_{\frac{t_{i}}{t}}^{1}(1-w)^{n-\alpha-1} w^{\alpha-1} d w\right]^{(n)}}{\Gamma(n-\alpha)} \\
+\frac{\left[-\int_{0}^{t}(t-u)^{n-1} \int_{0}^{1}(1-w)^{n-\alpha-1} \frac{w^{\alpha-1}}{\Gamma(\alpha)} d w h(u) d u\right]^{(n)}}{\Gamma(n-\alpha)} \\
-h(t)+\frac{\left[\sum_{\mu=0}^{i-1} M_{\mu+1} t^{n-1} \int_{\frac{t_{\mu}}{t}}^{\frac{t_{\mu+1}}{t}}(1-w)^{n-\alpha-1} w^{\alpha-1} d w\right]^{(n)}}{\Gamma(n-\alpha)} \\
+\frac{\left[M_{i+1} \int_{\frac{t_{i}}{t}}^{1}(1-w)^{n-\alpha-1} w^{\alpha-1} d w\right]^{(n)}}{\Gamma(n-\alpha)} .
\end{gathered}
$$

It is easy to see that $D_{0^{+}}^{\alpha} x(t) \neq-h(t)$ on $\left(t_{1}, t_{2}\right]$. In fact, we find that $D_{0^{+}}^{\alpha} x(t) \neq-h(t)$ on $\left(t_{1}, t_{2}\right]$ if and only if $M_{1}=M_{2}$.

\section{References}

[1] Agarwal, R., Hristova, S., O'Regan, D., Stability of solutions to impulsive Caputo fractional differential equations, Electron. J. Diff. Equ., 58(2016), 1-22.

[2] Ahmad, B., Nieto, J.J., Existence of solutions for impulsive anti-periodic boundary value problems of fractional order, Taiwan. J. Math., 15(2011), no. 3, 981-993.

[3] Bai, Z., Dong, X., Yin, C., Existence results for impulsive nonlinear fractional differential equation with mixed boundary conditions, Boundary Value Problems, 1(2016), 1-11.

[4] Belmekki, M., Nieto, J.J., Rodriguez-López, R., Existence of solution to a periodic boundary value problem for a nonlinear impulsive fractional differential equation, Electron. J. Qual. Theory Differ. Equ., 16(2014), 27 pages.

[5] Hilfer, R., Application of Fractional Calculus in Physics, World Scientific Publishing Company, Singapore, 2000.

[6] Kilbas, A.A., Srivastava, H.M., Trujillo, J.J., Theory and Applications of Frational Differential Equations, Elsevier Science B.V., Amsterdam, 2006.

[7] Kilbas, A.A., Trujillo, J.J., Differential equations of fractional order: methods, results and problems, I, Appl. Anal., 78(2001), 153-192.

[8] Kosmatov, N., Initial value problems of fractional order with fractional impulsive conditions, Results. Math., 63(2013), 1289-1310.

[9] Liu, Y., Studies on impulsive differential models with multi-term Riemann-Liouville fractional derivatives, Journal of Applied Mathematics and Computing, 522016, no. 1-2, 529-565.

[10] Liu, Y., On piecewise continuous solutions of higher order impulsive fractional differential equations and applications, Appl. Math. Comput., 287(2016), 38-49. 
[11] Liu, Y., Survey and new results on boundary-value problems of singular fractional differential equations with impulse effects, Electron. J. Differ. Equ, 16(2016), 296.

[12] Mawhin, J., Topological degree methods in nonlinear boundary value problems, in: NSFCBMS Regional Conference Series in Math., American Math. Soc. Providence, RI, 1979.

[13] Rehman, M., Eloe, P.W., Existence and uniqueness of solutions for impulsive fractional differential equations, Appl. Math. Comput., 224(2013), 422-431.

[14] Wang, H., Lin, X., Anti-periodic BVP of fractional order with fractional impulsive conditions and variable parameter, J. Appl. Math. Comput.

[15] Wang, J., Zhang, Y., On the concept and existence of solutions for fractional impulsive systems with Hadamard derivatives, Appl. Math. Letters, 39(2015), 85-90.

[16] Wang, J., Zhou, Y., Feckan, M., On recent developments in the theory of boundary value problems for impulsive fractional differential equations, Comput. Math. Appl., 64(10)(2012), 3008-3020.

[17] Zhao, K., Impulsive fractional differential equation higher order problems of the higherorder fractional differential equation with eigenvalue arguments, Adv. Differ. Equ., 382(2015) 16 pages.

Yuji Liu

Department of Mathematics and Statistics

Guangdong University of Finance and Economics

Guangzhou 510320, P.R. China

e-mail: liuyuji888@sohu.com 